\title{
MASP-2 Is a Heparin-Binding Protease; Identification of Blocking Oligosaccharides
}

\author{
Ditmer T. Talsma ${ }^{1}$, Felix Poppelaars ${ }^{1}$, Wendy Dam ${ }^{1}$, Anita H. Meter-Arkema ${ }^{1}$, \\ Romain R. Vivès ${ }^{2}$, Peter Gál ${ }^{3}$, Geert-Jan Boons ${ }^{4,5}$, Pradeep Chopra ${ }^{5}$, Annamaria Naggi ${ }^{6}$, \\ Marc A. Seelen ${ }^{1}$, Stephan P. Berger ${ }^{1}$, Mohamed R. Daha ${ }^{1}$, Coen A. Stegeman ${ }^{1}$, \\ Jacob van den Born ${ }^{1 *}$ and the COMBAT Consortium \\ ${ }^{1}$ Department of Nephrology, University Medical Center Groningen, Groningen, Netherlands, ${ }^{2}$ Univ. Grenoble Alpes, CNRS, \\ CEA, IBS, Grenoble, France, ${ }^{3}$ Institute of Enzymology, Research Centre for Natural Sciences, Budapest, Hungary, \\ ${ }^{4}$ Department of Chemical Biology and Drug Discovery, Utrecht Institute for Pharmaceutical Sciences, and Bijvoet Center for \\ Biomolecular Research, Utrecht University, Utrecht, Netherlands, ${ }^{5}$ Complex Carbohydrate Research Center, University of \\ Georgia, Athens, GA, United States, ${ }^{6}$ Ronzoni Institute, Milan, Italy
}

OPEN ACCESS

Edited by:

Megan S. Lord,

University of New South

Wales, Australia

Reviewed by:

Jeremy Turnbull,

University of Liverpool, United Kingdom

Douglas Philip Dyer, University of Manchester, United Kingdom

*Correspondence: Jacob van den Born j.van.den.born@umcg.nl

Specialty section: This article was submitted to Molecular Innate Immunity, a section of the journal

Frontiers in Immunology

Received: 09 October 2019 Accepted: 31 March 2020

Published: 28 April 2020

Citation:

Talsma DT, Poppelaars F, Dam W, Meter-Arkema AH, Vivès RR, Gál $P$,

Boons G-J, Chopra P, Naggi A, Seelen MA, Berger SP, Daha MR, Stegeman $C A$, van den Born $J$ and the COMBAT Consortium (2020) MASP-2 Is a Heparin-Binding Protease; Identification of Blocking Oligosaccharides.

Front. Immunol. 11:732. doi: 10.3389/fimmu.2020.00732 prevent the lectin pathway from activation.
It is well-known that heparin and other glycosaminoglycans (GAGs) inhibit complement activation. It is however not known whether fractionation and/or modification of GAGs might deliver pathway-specific inhibition of the complement system. Therefore, we evaluated a library of GAGs and their derivatives for their functional pathway specific complement inhibition, including the MASP-specific C4 deposition assay. Interaction of human MASP-2 with heparan sulfate/heparin was evaluated by surface plasmon resonance, ELISA and in renal tissue. In vitro pathway-specific complement assays showed that highly sulfated GAGs inhibited all three pathways of complement. Small heparin- and heparan sulfate-derived oligosaccharides were selective inhibitors of the lectin pathway $(\mathrm{LP})$. These small oligosaccharides showed identical inhibition of the ficolin-3 mediated LP activation, failed to inhibit the binding of MBL to mannan, but inhibited C4 cleavage by MASPs. Hexa- and pentasulfated tetrasaccharides represent the smallest MASP inhibitors both in the functional LP assay as well in the MASP-mediated C4 assay. Surface plasmon resonance showed MASP-2 binding with heparin and heparan sulfate, revealing high Kon and Koff rates resulted in a $\mathrm{Kd}$ of $\sim 2 \mu \mathrm{M}$ and confirmed inhibition by heparin-derived tetrasaccharide. In renal tissue, MASP-2 partially colocalized with agrin and heparan sulfate, but not with activated C3, suggesting docking, storage, and potential inactivation of MASP-2 by heparan sulfate in basement membranes. Our data show that highly sulfated GAGs mediated inhibition of all three complement pathways, whereas short heparin- and heparan sulfate-derived oligosaccharides selectively blocked the lectin pathway via MASP-2 inhibition. Binding of MASP-2 to immobilized heparan sulfate/heparin and partial co-localization of agrin/heparan sulfate with MASP, but not C3b, might suggest that in vivo heparan sulfate proteoglycans act as a docking platform for MASP-2 and possibly

Keywords: lectin pathway, MASP-2, tetrasaccharide, heparin, complement, glycosaminoglycans 


\section{INTRODUCTION}

As a part of the innate immune system, complement consists of soluble, and cell bound proteins. The complement system is activated via three different pathways; the classical pathway $(\mathrm{CP})$, lectin pathway (LP), and alternative pathway (AP). The $\mathrm{CP}$ is initiated by the binding of $\mathrm{Clq}$ to IgG or IgM and the LP by pattern recognition molecules binding to carbohydrates of pathogens or self-antigen. This leads to a conformational change and subsequent activation of the associated serine proteases $\mathrm{C} 1 \mathrm{r} / \mathrm{C} 1 \mathrm{~s}$ and MASP-1/MASP-2, respectively. These serine proteases cleave $\mathrm{C} 2$ and $\mathrm{C} 4$, forming the $\mathrm{C} 4 \mathrm{bC} 2 \mathrm{a}$ complex, a C3 convertase which deposits C3b initiating the amplification loop. The AP can be initiated either by auto activation of $\mathrm{C} 3$ eventually forming the $\mathrm{C} 3$ convertase $\mathrm{C} 3 \mathrm{bBb}$, or by in situ binding of AP stimulator properdin to the cell surface. Formation of the C5 convertase in the end leads to the generation of the C5b-9 membrane attack complex, resulting in cell lysis (1).

In the field of nephrology, complement has gained increased attention in recent years as studies have identified complement as a key player in multiple renal diseases. The classical pathway (CP) has been shown to play a major role in the autoimmune disease lupus erythematosus (2). In addition, lectin pathway (LP) components, either in plasma, or deposited within the kidney, have been correlated to disease progression following human kidney transplantation and hemodialysis, IgA nephropathy and diabetic nephropathy (3-6). Furthermore, it has been shown that mannan binding lectin (MBL) and collectin11 recognize epitopes in $\mathrm{I} / \mathrm{R}$ damaged kidneys and increase $\mathrm{I} / \mathrm{R}$ induced damage $(7,8)$. Finally, the alternative pathway (AP) has been identified as a factor in the physiopathology of dense deposit disease, C3 glomerulopathy, atypical hemolytic uremic syndrome, and progression of proteinuric renal diseases (9-13). Therefore, complement-targeted therapies can potentially be of great use in a variety of renal diseases and conditions.

The in vivo inhibitory potential of heparin on the complement system has been known for $\sim 25$ years (14). Since then, numerous interactions have been described between glycosaminoglycans (GAGs) such as heparin, and complement components. In the lectin route of complement, anti-thrombin bound to heparin is a strong inhibitor of C4 cleavage by MASPs (15). Besides the lectin pathway, heparin can also block the classical pathway by directly inhibiting the $\mathrm{Clq}$ subunit of $\mathrm{C} 1$ or by potentiating the effect of C1-inhibitor (16-18). Studies by our group showed that the binding of both properdin, an alternative pathway initiator and stabilizer, and factor $\mathrm{H}$, an alternative pathway inhibitor, to heparan sulfates (HS) on proximal tubular epithelial cells can be prevented by heparin and some other GAGs $(12,13)$. Combined, these studies indicate that GAGs have the potential to inhibit different components of the three pathways of the complement system.

Proteoglycans are glycoconjugates consisting of a core protein to which GAGs are covalently attached. Proteoglycans, such as the members of the syndecan and the glypican families, can be found on the cell membrane, others like versican, perlecan, and agrin are found in the extracellular matrix. Membrane proteoglycans function as highly abundant, relatively low affinity co-receptors for growth factors, chemokines, and adhesion molecules and modulate proliferation, migration, and adhesion events (19). Matrix-associated proteoglycans mostly function as storage depot for mediators, which can be released for paracrine functions upon tissue remodeling by proteases, sulfatases, and/or heparanase (20-22). GAGs consist of repetitive disaccharide units, which can be modified enzymatically in their length and sulfation pattern to alter their binding capacity and function. The effects on binding capacity and pharmacokinetics of GAG length modifications have been known for some time and have led to the introduction of low molecular weight heparins. Modifications of heparan sulfate (HS) sulfation are well-known from in vivo modifications of proteoglycan side chains upon stimuli like inflammation and fibrosis $(23,24)$. HS/Heparin can carry sulfate groups on the $\mathrm{N}$ position and/or on the $3-\mathrm{O}, 6-\mathrm{O}$, and $2-\mathrm{O}$ position of glucosamine and iduronic acid residues, respectively. Degree of sulfation is positively correlated to the electronegativity of HS/Heparin and therefore to their binding capacity, making it an important factor in various cellular functions. It has for example been shown that the binding of heparin to antithrombin III depends on a specific pentasaccharide structure, in which 3-O sulfation is essential (25). Moreover, our group has shown that properdin and factor $\mathrm{H}$ require different GAG sulfation patterns for HS binding (13). These examples illustrate the important role of chain length and sulfation pattern of GAGs for specific interactions related to biological properties.

In this study, we aimed at identifying pathway-specific complement inhibiting GAGs from a library of natural and enzymatically and chemically modified and/or depolymerized GAGs. We show that small heparin- and HS-derived oligosaccharides are specific inhibitors of the LP of complement and that these sugars inhibit the LP via inhibition of the MASP-2 enzyme. By surface plasmon resonance we confirm MASP-2 to be a HS/heparin binding protein. Moreover, we provide some evidence that HS proteoglycans in situ might bind and eventually regulate MASP-2 in vivo.

\section{MATERIALS AND METHODS}

\section{Polysaccharides}

Heparin from ovine intestinal mucosa, heparin from bovine lung, heparin $(\sim 2.5$ sulfate groups/disacch $)$ and HS from porcine intestinal mucosa $(\sim 1.0$ sulfate groups/disacch), Chondroitin sulfate-A, -B, -and C, dextran T40, dextran sulfate, fucoidan, were purchased from Sigma (Sigma, Zwijndrecht, The Netherlands). Nadroparin (Fraxiparine ${ }^{\circledR}$ ) was purchased from Sanofi Winthrop (Maassluis, The Netherlands), Dalteparin (Fragmin ${ }^{\circledR}$ ) was purchased from Pharmacia \& Upjohn, and enoxaparin $\left(\right.$ Clexane ${ }^{\circledR}$ ) was purchased from Rhone-Poulenc Rorer (Paris, France). HS isolated from bovine kidney $(\sim 0.8$ sulfate groups/disacch) or from Engelbreth-Holm-Swarm sarcoma ( $\sim 0.6$ sulfate groups/disacch) were obtained from Seikagaku Corp (Tokyo, Japan). Escherichia coli capsular polysaccharide K5, with the same (GlcUA $\rightarrow$ GlcNAc) ${ }_{n}$ structure as the non-sulfated HS/heparin biosynthetic precursor polysaccharide ( 0.0 sulfate groups/disacch) (26); $\mathrm{O}$-sulfated $\mathrm{K} 5$ and low molecular weight $\mathrm{O}$-sulfated $\mathrm{K} 5$; were kindly provided 
by Dr. G. van Dedem (Diosynth, Oss, The Netherlands), as well as the heparin-derived octasaccharides (Org32100), hexasaccharides (Org 32101), and tetrasaccharides (Org 32102). HS from bovine intestine was kindly provided by Marco Maccarana (Department of Experimental Medical Science, Biomedical Center, University of Lund, Sweden) (27). HS from human aorta was isolated essentially as described by Iverius $(\sim 0.6$ sulfate groups/disacch) (28). $\mathrm{N}+\mathrm{O}$-sulfated $\mathrm{K} 5$ was produced by $N$-deacetylation (hydrazinolysis), subsequent $N$-sulfation with sulfur trioxide-trimethylamine (29) of O-sulfated K5, followed by $\mathrm{N}$-acetylation. Heparin and HS-derived oligosaccharides were prepared as previously described $(30,31)$ by partial heparinase I (Grampian enzymes, Orkney, UK) depolymerization of porcine mucosal heparin and exhaustive digestion of porcine mucosal HS with heparinase III (Grampian enzymes). Disaccharide analysis of SAGAG heparin tetrasaccharides was achieved by reverse-phase ion-pair high-performance liquid chromatography (RPIP-HPLC) as described before (32). Treatment of heparin by the 6-O-endosulfatase HSulf-2 was performed as described previously (33), for details on the disaccharide composition, see (34). Enoxaparin tetrasaccharides (Ron G11237) were kindly provided by dr. Annamaria Naggi (Ronzoni Institute, Milan, Italy) isolated and characterized by NMR spectroscopy as described before (35), N-desulfated, re-acetylated heparin and periodate-oxidized and reduced heparin were also provided by dr. Annamaria Naggi and were prepared and characterized as described before (36). Synthetic HS tetrasaccharides were kindly provided by prof. dr. G.J.P.H. Boons (Pharmacy, State University of Utrecht, The Netherlands) and were produced as described before (37).

\section{Wieslab Pathway-Specific Complement Assay}

The Wieslab complement assay (WieLISA) kits were obtained from Euro Diagnostica, Malmö, Sweden. The WieLISA assay is a straightforward ELISA-based format for the evaluation of the three pathways of complement activation. The assays are based on specific coatings for each pathway in combination with specific buffer systems and measure the deposition of the terminal C5b-9 MAC complex. The measurement of the lectin pathway is either done by using mannan coated plates, allowing binding of the MBL-MASP complex from serum (in the standard WieLISA assay), or by using acetylated BSA coated plated, allowing binding of ficolin-3-MASP complexes from serum (in the ficolin-3 LP assay). The assays have been described by Seelen et al. (38). Positive control serum delivered with the kits was used as serum source for all measurements. GAGs were diluted in route specific buffer delivered with the kit. Final GAG concentration was $100 \mu \mathrm{g} / \mathrm{ml}$ in the CP and LP assay and due to a higher incubated serum concentration $200 \mu \mathrm{g} / \mathrm{ml}$ in the AP assay. Serum was added to the diluted GAGs right before the plates were incubated at $37^{\circ} \mathrm{C}$ for $60 \mathrm{~min}$. Except for the serum $(+/-$ GAGs) incubation, the kit protocol was followed. Data were expressed as \% inhibition compared to the positive control. Dose dependent assays with variable amounts of GAGs were performed as described above. Data was presented as representative experiments.

\section{MBL Binding Assay}

To evaluate whether GAGs could inhibit the mannan-MBL interaction, Maxisorp immunoassay plates were incubated overnight with $100 \mu \mathrm{g} / \mathrm{ml}$ mannan (Sigma, Zwijndrecht, The Netherlands) diluted in $0,1 \mathrm{M} \mathrm{NaCO}_{3}, \mathrm{pH} 9,6$. After washing three times, plates were blocked with $1 \%$ BSA in PBS for $1 \mathrm{~h}$. Thereafter, pooled serum diluted 1:50 in GVB++ buffer, described by Roos et al. (39), were pre-incubated with a concentration range of GAGs, for $15 \mathrm{~min}$ at room temperature. $\mathrm{GVB}++$ buffer is Veronal-buffered saline $(1,8 \mathrm{mM} \mathrm{Na}-5,5-$ diethylbarbital, 0,2 $\mathrm{mM}$ 5,5-diethylbarbituric acid, $145 \mathrm{mM}$ $\mathrm{NaCl}$ ) containing 0,5 mM MgCl2, $2 \mathrm{mM} \mathrm{CaCl} 2,0,05 \%$ Tween-20 and $0,1 \%$ gelatin, $\mathrm{pH} 7.5$. The pre-incubated mixture was then incubated for $60 \mathrm{~min}$ on the mannan coated plates at $37^{\circ} \mathrm{C}$. In the dose dependent binding of MBL to mannan, no GAGs were added. Bound MBL was detected with a DIG-labeled mouse antihuman MBL antibody 1:1,000 (mAb 3e7 from Hycult Biotech, Uden, the Netherlands) and a Sheep anti-DIG HRP labeled conjugate 1:8,000 (Roche Diagnostics, Mannheim, Germany). The assay was developed using tetramethylbenzidine (TMB) (Sigma, Zwijndrecht, The Netherlands) and the reaction was stopped with $1 \mathrm{M} \mathrm{H} 2 \mathrm{SO} 4$. Absorbance was measured at $450 \mathrm{~nm}$ in a microplate reader. Data was expressed as \% inhibition compared to non-inhibited control. Data was presented as representative experiment.

\section{C4 Inhibition Assays}

To evaluate whether GAGs inhibit the C4 cleavage (by C4d deposition) by MASPs, we performed a C4 cleaving assay, as first described by Petersen and colleagues (40). Maxisorp immunoassay plates were incubated overnight with $100 \mu \mathrm{g} / \mathrm{ml}$ mannan (Sigma, Zwijndrecht, The Netherlands) diluted in 0,1 M $\mathrm{NaCO}_{3}, \mathrm{pH} 9,6$. Thereafter, plates were blocked for $1 \mathrm{~h}$ using $10 \mathrm{mM}$ Tris- $\mathrm{HCl}, 140 \mathrm{mM} \mathrm{NaCl}, 0,1 \%$ BSA, pH 7,4. Next, pooled serum diluted $1: 100$ in $20 \mathrm{mM}$ Tris- $\mathrm{HCl}, 10 \mathrm{mM} \mathrm{CaCl}_{2}, 1 \mathrm{M}$ $\mathrm{NaCl}, 0,05 \%$ Triton X-100, 0,1\% BSA, pH 7,4 was incubated overnight at $4^{\circ} \mathrm{C}$, allowing the MBL/MASP complex to bind to mannan, but prevents MASP activation and subsequent complement activation. Incubation of serum in $1 \mathrm{M} \mathrm{NaCl}$ was done to prevent classical pathway activation through antimannan IgG3 antibodies, prevalent in the majority of the healthy population. High ionic buffers prevent the binding of $\mathrm{C} 1 \mathrm{q}$ to immune complexes and disrupt the $\mathrm{C} 1$ complex, whereas the carbohydrate-binding activity of $\mathrm{MBL}$ and the integrity of the MBL complex are maintained under hypertonic conditions. After washing, MBL/MASP coated plates were pre-incubated with 50 $\mu l \mathrm{GAG}$ at twice the final concentration in $10 \mathrm{mM}$ Tris- $\mathrm{HCl}$, $140 \mathrm{mM} \mathrm{NaCl}, 5 \mathrm{mM} \mathrm{CaCl}$, 0,05\% Tween, 0,1\% BSA, pH 7,4 for $30 \mathrm{~min}$ at $37^{\circ} \mathrm{C}$. Non-inhibited control wells were incubated with buffer only. Without washing, $50 \mu \mathrm{l}$ of $5 \mu \mathrm{g} / \mathrm{ml}$ purified C4 (Hycult Biotech, Uden, the Netherlands) diluted in $10 \mathrm{mM}$ Tris$\mathrm{HCl}, 140 \mathrm{mM} \mathrm{NaCl}, 5 \mathrm{mM} \mathrm{CaCl}$, 0,05\% Tween, 0,1\% BSA, pH 7,4 was added to the pre-incubated GAGs and incubated for $1 \mathrm{~h}$ at $37^{\circ} \mathrm{C}$. After washing, C4d deposition was detected using a DIG 
labeled mouse anti-human C4 antibody, diluted 1:4,000, followed by incubation with a Sheep anti-DIG HRP labeled antibody (Roche Diagnostics, Mannheim, Germany, dilution 1:8,000). The assay was developed using TMB (Sigma, Zwijndrecht, The Netherlands) and the reaction was stopped with $1 \mathrm{M}$ $\mathrm{H}_{2} \mathrm{SO}_{4}$. Absorbance was measured at $450 \mathrm{~nm}$ in a microplate reader. Data was expressed as \% inhibition compared to noninhibited control. Experiments were independently reproduced in triplicate.

\section{Heparin-MBL/MASP Interaction ELISA}

In three independent experiments, we tested the binding of serum MASP-2 and recombinant MASP2 to heparin. To this end, we coated Maxisorp immunoassay plates overnight with $5 \mu \mathrm{g} / \mathrm{ml}$ heparin-albumin or $5 \mu \mathrm{g} / \mathrm{ml}$ albumin diluted in PBS. Heparin-albumin was from Sigma-Aldrich (Saint Louis, MO, USA). According to the data sheet, this artificial proteoglycan contained 4.8 moles heparin per mole albumin, protein content is about 55\%. After washing, plates were blocked using 1\% BSA in PBS for $1 \mathrm{~h}$. Thereafter, recombinant MASP2 or pooled human serum diluted in GVB++ buffer was incubated for $2 \mathrm{~h}$ at $4^{\circ} \mathrm{C}$. Binding of MASP-2 was detected by incubating rat anti-human MASP-2 (Hycult Biotech, Uden, the Netherlands) diluted at 1:200 in PBS, $1 \%$ BSA and 0,05\% Tween. Rabbit anti-Rat HRP labeled (DAKO, Glostrup, Denmark) diluted 1:500 in PBS, 1\% BSA and $0,05 \%$ Tween was incubated for $1 \mathrm{~h}$. The assay was developed using TMB (Sigma, Zwijndrecht, The Netherlands), the reaction was stopped with $1 \mathrm{M} \mathrm{H}_{2} \mathrm{SO}_{4}$. Absorbance was measured at $450 \mathrm{~nm}$ in a microplate reader.

\section{Surface Plasmon Resonance Analysis}

To define the binding affinity of MASP-2 to heparin derivatives surface plasmon resonance (SPR) experiments were performed using recombinant human MASP-2 protein containing the catalytic fragment (CCP1-CCP2-SP), which was prepared as described earlier (41). All experiments were performed on a BIAcore T200 (GE healthcare), using as previously described standard procedures and GAG biotinylation techniques (40). Briefly, reducing-end biotinylated 6 and $15 \mathrm{kDa}$ heparin, and HS (porcine mucosa) were captured on three streptavidin-activated flowcells of a S-CM4 sensorchip (41.2 and 44.1 RU for 6 and $15 \mathrm{kDa}$ heparin respectively, and $61.9 \mathrm{RU}$ for $\mathrm{HS}$, a fourth one being used as negative control surface. Using HBS-P+ running buffer (10 mM HEPES, $150 \mathrm{mM} \mathrm{NaCl}, 0.05 \%$ surfactant P20, $\mathrm{pH} 7.4$ ) at a flow rate of $20 \mu \mathrm{l} / \mathrm{min}$, series of $0-3,000 \mathrm{nM}$ of MASP-2 were injected over the heparin, HS and negative control surfaces, followed by a 3 min washing step with HBS$\mathrm{P}+$ buffer to allow dissociation of the complexes formed. At the end of each cycle, surfaces were regenerated with a $2.5 \mathrm{~min}$ injection of $2 \mathrm{M} \mathrm{NaCl}$. Sensorgram shown correspond to online subtraction of the negative control to the heparin surface signal. Kds were determined using the BIAcore T200 evaluation (version 3.1) software, by steady state analysis (1:1 binding model). For this, resonance values (Req) were taken within the equilibrium phase (just before the end of injection) and plotted against MASP- 2 concentration. Calculated $\chi 2$ values provided quality control of experimental data fitting. Competition assays were performed similarly, by injecting $200 \mathrm{nM}$ of MASP-2 preincubated with GAGs $(30 \mu \mathrm{g} / \mathrm{ml})$ over the heparin and negative control surfaces. Tested GAGs included a heparin tetrasaccharide ( $\triangle$ HexA2S-GlCNS6S-IdoA2S-GlcNS6S) and chondroitin sulfate $\mathrm{A}, \mathrm{B}$ and $\mathrm{C}$, the $15 \mathrm{kDa}$ heparin being used as a positive control (also $30 \mu \mathrm{g} / \mathrm{ml}$, a 10 -fold excess compared to the concentration used to load the sensor chip). Noteworthy, this experiments was conducted using fixed tetrasaccharide and heparin mass concentrations to reflect the fact that a $15 \mathrm{kDa}$ heparin chain comprises multiple highly sulfated tetrasaccharide (composition of $15 \mathrm{kDa}$ Heparin is provided in Seffouh et al. (42). All experiments were independently replicated three times.

\section{Immunohistochemistry}

Four $\mu \mathrm{m}$ frozen kidney sections of five human donor kidneys not suitable for transplantation for anatomical reasons were used for immunofluorescent stainings. Sections were double stained for MASP-2 in combination with the basement membrane HS proteoglycan agrin, or in combination with HS (clone $10 \mathrm{E} 4$ recognizing mixed $\mathrm{N}$-sulfated/N-acetylated sequences) or triple stained with $\mathrm{HS}$ and activated complement factor C3 (recognizing a neoepitope on $\mathrm{C} 3 \mathrm{~b}, \mathrm{iC} 3 \mathrm{~b}$, and $\mathrm{C} 3 \mathrm{c}$ after cleavage of C3 by C3-convertase). Details of the immunofluorescence procedures and the antibodies used are given in Table 1. Photomicrographs were taken at $630 \mathrm{x}$ magnification with confocal microscope (Leica SP8, Leica microsystems BV, Rijswijk, the Netherlands) at the Imaging and Microscopy Center of the University Medical Center Groningen.

\section{RESULTS}

\section{Small Heparan Sulfate- and Heparin-Derived Oligosaccharides Specifically Inhibit the Lectin Pathway, While Longer Highly Sulfated GAGs Block All Three Routes of Complement}

A library of naturally, chemically, or enzymatically modified and synthetic GAGs, as well as size-defined HS/heparin depolymerization products were tested for their complement inhibiting potential in the WieLISA, which allows separate evaluation of the three pathways of the complement system. From the results (Table 2), it can be seen that unfractionated heparins inhibited all three complement pathways. Heparin from different sources e.g., porcine intestinal mucosa and bovine lung showed strong inhibitory potential for all pathways.

Selective desulfation of heparin, either 6-O desulfation by SULF2 (80\% reduction of the 6-O content in NS2S6S disaccharides), or by chemical $\mathrm{N}$-desulfation followed by reacetylation, resulted in some loss of complement inhibitory potential, mostly in the classical and alternative route.

Periodate-oxidized and reduced heparin is a nonanticoagulant heparin derivate in which the structure of the antithrombin binding site was modified, and the results in Table 2 show that despite losing the ability to interact with antithrombin III, it remained able to inhibit all complement pathways. LMW-heparins like fragmin, fraxiparin and 
TABLE 1 | Details on immunofluorescence stainings on human cryosections.

\begin{tabular}{|c|c|c|c|c|}
\hline Procedure & MASP-2 & Agrin & Heparan sulfate & Activated C3 \\
\hline Fixation & $\begin{array}{l}\text { Icecold } 100 \% \text { aceton for } \\
10 \mathrm{~min}\end{array}$ & $\begin{array}{l}\text { Icecold } 100 \% \text { aceton for } \\
10 \mathrm{~min}\end{array}$ & $\begin{array}{l}\text { Icecold } 100 \% \text { aceton for } \\
10 \mathrm{~min}\end{array}$ & $\begin{array}{l}\text { Icecold } 100 \% \text { aceton for } 10 \\
\text { min }\end{array}$ \\
\hline Peroxidase inactivation & $\begin{array}{l}0.03 \% \mathrm{H}_{2} \mathrm{O}_{2} \text { in PBS for } \\
30 \text { min in the dark }\end{array}$ & - & - & - \\
\hline $\begin{array}{l}\text { Blocking a-specific } \\
\text { background }\end{array}$ & $1 \% \mathrm{BSA}$ in PBS for $15 \mathrm{~min}$ & $1 \% \mathrm{BSA}$ in PBS for $15 \mathrm{~min}$ & $1 \%$ BSA in PBS for 15 min & $1 \%$ BSA in PBS for 15 min \\
\hline Blocking endogenous biotin & - & - & $\begin{array}{l}\text { Avidin/Biotin blocking kit } \\
\text { (SP-2001; Vector } \\
\text { Laboratories; Burlingame } \\
\text { CA, USA) }\end{array}$ & - \\
\hline Primary antibody & $\begin{array}{l}\text { Rat mAb anti-human } \\
\text { MASP-2, clone 8B5, 1:100 } \\
\text { (Hycult, Uden, The } \\
\text { Netherlands) }\end{array}$ & $\begin{array}{l}\text { Mouse mAb JM-72 } \\
\text { anti-human agrin, 1:750 (43) }\end{array}$ & $\begin{array}{l}\text { Biotinylated mouse mAb } \\
\text { anti-heparan sulfate, clone } \\
\text { 10E4, 1:50 (Amsbio, } \\
\text { Abingdon, UK) }\end{array}$ & $\begin{array}{l}\text { Mouse IgG2a mouse mAb } \\
\text { anti-neoepitope on C3b, } \\
\text { ic3b and C3c, clone bH6, } \\
\text { 1:200 (Hycult) }\end{array}$ \\
\hline Secondary antibody & $\begin{array}{l}\text { Rabbit anti-rat lgG-HRP, } \\
1: 200+5 \% \text { normal human } \\
\text { serum for (Dako, Glostrup, } \\
\text { Denmark) }\end{array}$ & $\begin{array}{l}\text { Donkey anti-Mouse } \\
\text { lgG-Alexa 488, 1:250 (Life } \\
\text { Technologies, Carlsbad CA, } \\
\text { USA) }\end{array}$ & - & $\begin{array}{l}\text { Donkey anti-mouse } \\
\text { lgG-Alexa647, 1:250 (Life } \\
\text { Technologies, Carlsbad CA, } \\
\text { USA) }\end{array}$ \\
\hline Tertiary antibody & $\begin{array}{l}\text { Goat anti-rabbit IgG-HRP, } \\
1: 200+5 \% \text { normal human } \\
\text { serum (Dako) }\end{array}$ & - & - & - \\
\hline Amplification HRP signal & $\begin{array}{l}\text { TSA Tyramide-TRITC, 1:50 } \\
\text { for } 10 \text { min (PerkinElmer, } \\
\text { Waltham MA, USA) }\end{array}$ & - & - & - \\
\hline $\begin{array}{l}\text { Detection biotinylated } \\
\text { antibody }\end{array}$ & - & - & $\begin{array}{l}\text { Streptavidin-FITC, 1:300 } \\
\text { (Invitrogen, Waltham MA, } \\
\text { USA) }\end{array}$ & - \\
\hline Nuclear staining & Dapi for $10 \mathrm{~min}$ & Dapi for $10 \mathrm{~min}$ & Dapi for $10 \mathrm{~min}$ & Dapi for 10 min \\
\hline Embedment & Citifluor (Haffield PA, USA) & Citifluor (Haffield PA, USA) & Citifluor (Haffield PA, USA) & Citifluor (Haffield PA, USA) \\
\hline
\end{tabular}

Stainings are performed as MASP2/agrin double staining, as MASP2/HS double staining, and as MASP2/HS/actC3 triple staining.

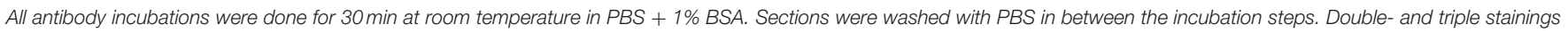
were designed in such a way that all cross-reactions among the stainings were avoided.

enoxaparin are widely used in the clinic as anticoagulants and showed strong potential to inhibit the three complement pathways. N-desulfation followed by reacetylation of LMW heparin reduced the ability to inhibit all complement pathways, mostly the classical pathway. Size fractionation products of limited heparinase I treated heparin showed that smaller heparin fragments retain their ability to inhibit the LP, but were no longer able to inhibit the CP and AP, indicating that GAG chain length is a major determinant for $\mathrm{CP}$ and AP inhibition. The smallest heparin fragment tested, i.e., tetrasaccharides (4-mer), were found to be completely selective for inhibition of the LP.

Escherichia coli-derived K5 polysaccharides share their polysaccharide backbone with heparin except that K5 polysaccharides carry exclusively GlcA uronic acid epimers, while heparin features $>80 \%$ IdoA C5 uronic epimers. Native $\mathrm{K} 5$ does not contain any sulfate groups and did not inhibit the complement system. O-sulfated and $\mathrm{N}+\mathrm{O}$-sulfated $\mathrm{K} 5$ both showed equally strong inhibitory capacity for all three complement pathways, indicating that neither GlcA to IdoA conversion nor $\mathrm{N}$-sulfation (next to $\mathrm{O}$-sulfation) is crucial for complement inhibition, when the density of $\mathrm{O}$-sulfates is high enough ( 1,5 O-sulfates/disacch in O-sulfated $\mathrm{K} 5)$. O-sulfated
K5 hexasaccharide did not inhibit the complement unlike heparin hexasaccharides (see above). Whether this is due to different positioning of the O-sulfate groups is unknown, but suggestive, which in regular heparin is mostly IdoA2S-GlcNs6S, and in chemically O-sulfated $\mathrm{K} 5$ mostly at $\mathrm{C} 2$ and $\mathrm{C} 3$ of GlcA along with $\mathrm{N}$-sulfation of Glc units.

We also tested GAGs and polysaccharides with a different backbone structure than heparin. These results indicate that the chemical nature of the monosaccharides and their bonds between the monosaccharide units is of lesser importance compared to the degree of sulfation when looking at complement inhibitory potential. Highly sulfated polysaccharides like fucoidan and dextran sulfate and to a lesser extent sulodexide [mixture of heparin $(80 \%)$ and dermatan sulfate $(20 \%)$ ] showed inhibition of all complement pathways. Chondroitin sulfate B (dermatan sulfate) with an intermediate amount of sulfates and high content of iduronic acid showed some inhibition of the LP, but not of the $\mathrm{CP}$ and $\mathrm{AP}$, while chondroitin sulfates $-\mathrm{A}$ and $-\mathrm{C}$ and the nonsulfated dextran T40 didn't inhibit the three complement routes.

HS have a slightly different disaccharide composition compared to heparin and are less and more variably sulfated (25, 44). HS from different sources (e.g., bovine kidney and porcine 
TABLE 2 | Complement inhibition by heparin(oids), (derivatives of) K5 polysaccharide, (derivatives of) heparan sulfates, and some other glycosaminoglycans.

\begin{tabular}{|c|c|c|c|c|}
\hline Glycosaminoglycans & $\begin{array}{l}\text { Classical } \\
\text { pathway }\end{array}$ & $\begin{array}{l}\text { Lectin } \\
\text { pathway }\end{array}$ & $\begin{array}{l}\text { Alternative } \\
\text { pathway }\end{array}$ & Repeats \\
\hline \multicolumn{5}{|c|}{ HEPARINS AND HEPARIN DERIVATIVES } \\
\hline Porcine intestinal mucosa & $73(12)$ & $97(1)$ & $94(5)$ & 2 \\
\hline Ovine intestinal mucosa & 83 & 97 & 96 & 1 \\
\hline Bovine lung & 66 & 90 & 74 & 1 \\
\hline SULF-2 treated heparin & 54 & 96 & 48 & 1 \\
\hline N-desulfated, re-acetylated heparin & 6 & 52 & 42 & 1 \\
\hline $\begin{array}{l}\text { Periodate-oxidized and reduced } \\
\text { heparin }\end{array}$ & 60 & 91 & 97 & 1 \\
\hline LMW-heparin (Fragmin) Mw: 6000 & 73 & 98 & 98 & 1 \\
\hline LMW-heparin (Fraxiparin) Mw: 4500 & 48 & 95 & 92 & 1 \\
\hline $\begin{array}{l}\text { LMW-heparin (Enoxaparin) Mw: } \\
4500\end{array}$ & 65 & 97 & 95 & 1 \\
\hline $\begin{array}{l}\text { LMW N-desulfated, reacetylated } \\
\text { heparin }\end{array}$ & 8 & 46 & 44 & 1 \\
\hline Heparin-derived 18-mer & 13 & 71 & 20 & 1 \\
\hline Heparin-derived 16-mer & 27 & 90 & 25 & 1 \\
\hline Heparin-derived 14-mer & 26 & 92 & 35 & 1 \\
\hline Heparin-derived 12-mer & 26 & 94 & 38 & 1 \\
\hline Heparin-derived 10-mer & 14 & 87 & 21 & 1 \\
\hline Heparin-derived 8-mer & 20 & 93 & 26 & 1 \\
\hline Heparin-derived 6-mer & 12 & 93 & 0 & 1 \\
\hline Heparin-derived 4-mer & 0 & 74 & 0 & 1 \\
\hline \multicolumn{5}{|c|}{ E. coli K5-DERIVED POLYSACCHARIDES } \\
\hline Native K5 & 0 & 1 & 3 & 1 \\
\hline O-sulfated K5 & $100(0)$ & $88(4)$ & $92(3)$ & 3 \\
\hline $\mathrm{N}-+$ O-sulfated K5 & 100 & 93 & 97 & 1 \\
\hline O-sulfated K5 hexasaccharides & 0 & 2 & 0 & 1 \\
\hline \multicolumn{5}{|l|}{ GLYCOSAMINOGLYCANS } \\
\hline Chondroitin sulfate A & $3(4)$ & $6(7)$ & $8(9) 2$ & 3 \\
\hline Chondroitin sulfate C & $0(0)$ & $0(0)$ & $0(0)$ & 2 \\
\hline $\begin{array}{l}\text { Chondroitin sulfate B (Dermatan } \\
\text { sulfate) }\end{array}$ & $5(7)$ & $39(5)$ & $10(10)$ & 3 \\
\hline Sulodexide & 52 & 93 & 73 & 1 \\
\hline \multicolumn{5}{|l|}{ POLYSACCHARIDES } \\
\hline Dextran T40 & 4 & 8 & 17 & 1 \\
\hline Dextran sulfate & 100 & 99 & 100 & 1 \\
\hline Fucoidan & 98 & 78 & 79 & 1 \\
\hline \multicolumn{5}{|c|}{ HEPARAN SULFATE AND HEPARAN SULFATE DERIVATIVES } \\
\hline HS human aorta & 5 & 23 & 7 & 1 \\
\hline HS EHS mouse sarcoma & 17 & 31 & 22 & 1 \\
\hline HS bovine intestine & 21 & 76 & 39 & 1 \\
\hline HS bovine kidney & $2(1)$ & $73(0)$ & $18(18)$ & 2 \\
\hline HS porcine mucosa & 9 & 95 & 26 & 1 \\
\hline Heparan sulfate derived 18-mer & 5 & 91 & 25 & 1 \\
\hline Heparan sulfate derived 16-mer & 11 & 92 & 32 & 1 \\
\hline Heparan sulfate derived 14-mer & 4 & 92 & 18 & 1 \\
\hline Heparan sulfate derived 12-mer & 0 & 93 & 3 & 1 \\
\hline Heparan sulfate derived 10-mer & 0 & 87 & 0 & 1 \\
\hline Heparan sulfate derived 8-mer & 0 & 74 & 0 & 1 \\
\hline Heparan sulfate derived 4-mer & 0 & 0 & 0 & 1 \\
\hline
\end{tabular}

A library of GAG-derived polysaccharides was tested in the WieLISA for their complement inhibiting potential. Values are expressed as percentage inhibition compared to control values without inhibitor as mean $\pm S D$ or from a single experiment. GAGs were added in a concentration of $100 \mathrm{ug} / \mathrm{ml}$ in the $C P$ and LP assay and due to a higher serum concentration at $200 \mathrm{ug} / \mathrm{ml}$ in the AP assay. mucosa) showed in general substantial LP inhibition without inhibiting the $\mathrm{CP}$ and AP. Smaller HS fragments were found to be more specific inhibitors of the LP. 12-mer, 10-mer and 8-mer HS fragments showed complete specificity for the LP and did not show any inhibition of the CP and AP (Figures 1A,C,D). The HS-derived 4-mer did not show any inhibitory capacity for any pathway (HS-derived 6-mer was not available) in contrast to heparin-derived tetrasaccharide, probably because of a lower degree of sulfation (Table 2).

To test the effect of GAG length on the inhibitory potential of the complement system, 5 heparin fragments ranging from unfractionated heparin to heparin-derived tetrasaccharides were tested in the WieLISA in a dose dependent fashion. As expected, unfractionated heparin showed the strongest inhibition in all pathways (Figures 1A-C). Interestingly, the LP was inhibited most potently by unfractionated heparin with an IC50 value of $2 \mu \mathrm{g} / \mathrm{ml}$ in 100-times diluted serum, in contrast to the CP (IC50: $39 \mu \mathrm{g} / \mathrm{ml}$ ) and the AP (IC50: $76 \mu \mathrm{g} / \mathrm{ml}$ ) (Figure 1D). Heparin octasaccharides and smaller heparin fragments become, up to the concentrations tested, specific LP inhibitors; IC50: octasaccharides: $3 \mu \mathrm{g} / \mathrm{ml}$, hexasaccharides: $4 \mu \mathrm{g} / \mathrm{ml}$ and tetrasaccharides: $21 \mu \mathrm{g} / \mathrm{ml}$ (Figure 1D). These results indicate that a certain heparin chain length is required for inhibition of the $\mathrm{CP}$ and $\mathrm{AP}$, while heparin fragments down to 4 saccharides in length can inhibit the LP.

To illustrate the effect of heparin/HS sulfation on the inhibitory capacity of complement, we selected 6 heparin/HS/K5 preparations with different sulfation degrees from Table 1 and displayed them in Figure 1E. Heparin with $>2,5$ sulfate groups per disaccharide showed, as observed before, strong inhibitory potential for all complement pathways. A reduced number of sulfate groups to 1,0 or 0,8 per disaccharide, attenuated predominantly the ability to inhibit the $\mathrm{CP}$ and AP. Further lowering the sulfate content resulted in a reduced inhibition of all complement pathways (Figure 1E).

Altogether, all three complement pathways can be blocked by highly sulfated polysaccharides such as heparin, fucoidan, dextran sulfate and O-sulfated K5 and to a lesser extent by some HS preparations and dermatan sulfate. Specific complement inhibition of the LP is achieved by some HS preparations and small heparin- and HS-derived oligosaccharides.

\section{Heparin Oligosaccharides Inhibit the Proteolytic Activity of MASPs and Thereby Reduce C4d Deposition}

The previous results showed that HS/heparin-derived oligosaccharides can specifically inhibit the LP of complement. The LP differs from the $\mathrm{CP}$ only in the pattern recognition molecule: MBL (in the WieLISA) vs. C1q, and the serine proteases, MASP- 1 and-2 vs. C1r and C1s. To pinpoint whether the small heparin oligosaccharides interfere with MBL or MASPs, the inhibitory effect of heparin (fragments) on the MBL-mannan interaction was tested. Serum was co-incubated with or without the heparin (fragments) on a mannan coated plate and MBL binding to mannan was used as a read out. MBL binding to mannan in the absence of inhibitors showed a dose 


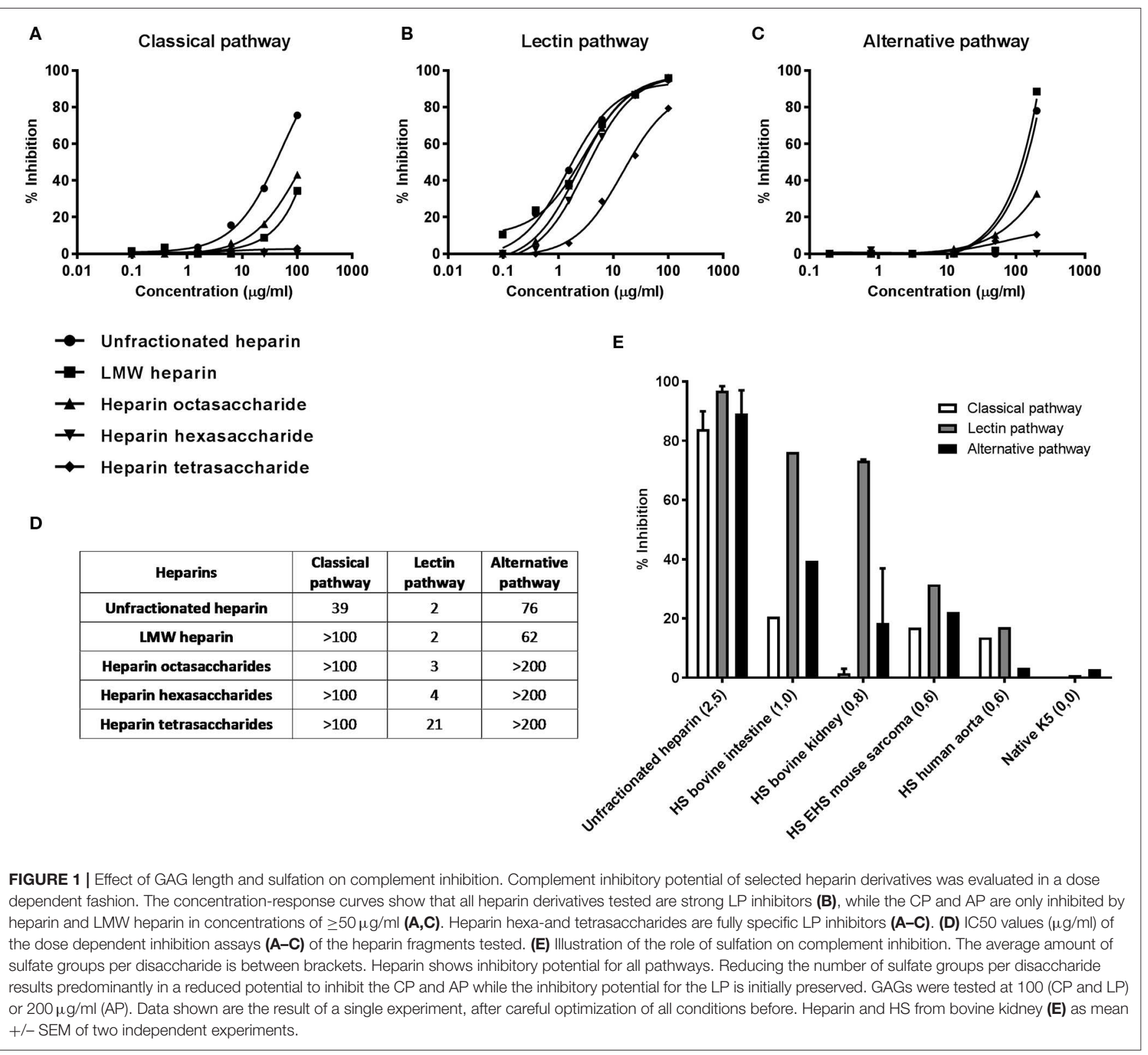

dependent binding, a serum concentration of 1:50 was used for the inhibition experiments (OD: 0.96) (Figure 2A). The results revealed that none of the selected heparin preparations, which all inhibit the LP in the WieLISA, could inhibit the MBL binding to mannan in any of the concentrations tested (Figure 2B). To strengthen the conclusion that heparin did not interfere with the MBL-mannan interaction, heparin (fragments) were tested in a ficolin-3 mediated LP activity assay. This assay measures LP activity with ficolin-3 as pattern recognition molecule for immobilized acetylated BSA instead of MBL with immobilized mannan. In ficolin-3 mediated LP activation cleaving of C4 and $\mathrm{C} 2$ is, like in the MBL mediated route, dependent on MASP activity (45). The heparin (fragments) showed a dose dependent inhibitory pattern in the ficolin-3 mediated LP assay identical to the MBL mediated WieLISA (Figure 2C vs. Figure 1B). Unfractionated heparin showed the strongest inhibitory effect with an IC50 of $3 \mu \mathrm{g} / \mathrm{ml}$. Decreasing the GAG length resulted in reduced inhibitory potential for LMW heparin, octasaccharides, hexasaccharides, and tetrasaccharides heparin (IC50: 7, 11, 16, and $342 \mu \mathrm{g} / \mathrm{ml}$, respectively). Since the LP and CP differ only in their pattern recognition molecules and their serine proteases and we showed that the heparin (oligoaccharides) are unable to inhibit the binding of pattern recognition molecules to pathogen associated molecular patterns, we suggest, based on these data, that the LP is inhibited by heparin (oligosaccharides) via the serine proteases, the MASP enzymes.

To prove this assumpsion, we next used an assay to measure the C4 cleavage potential (by measuring C4d deposition) 
A

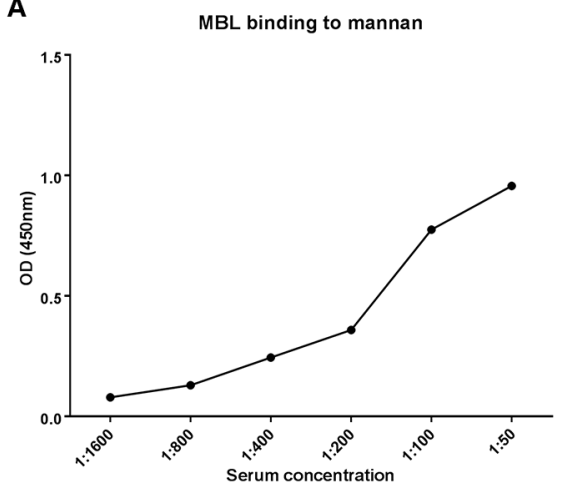

C

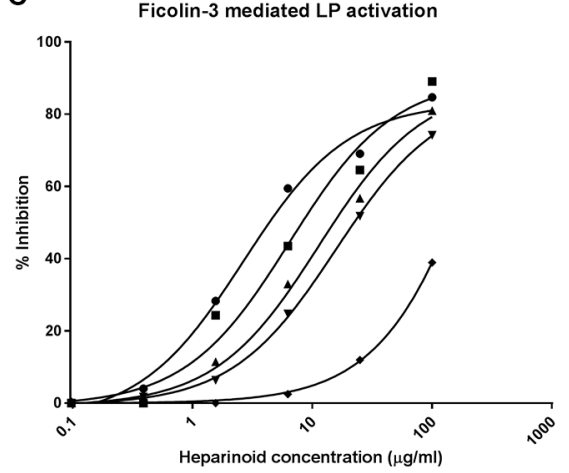

B
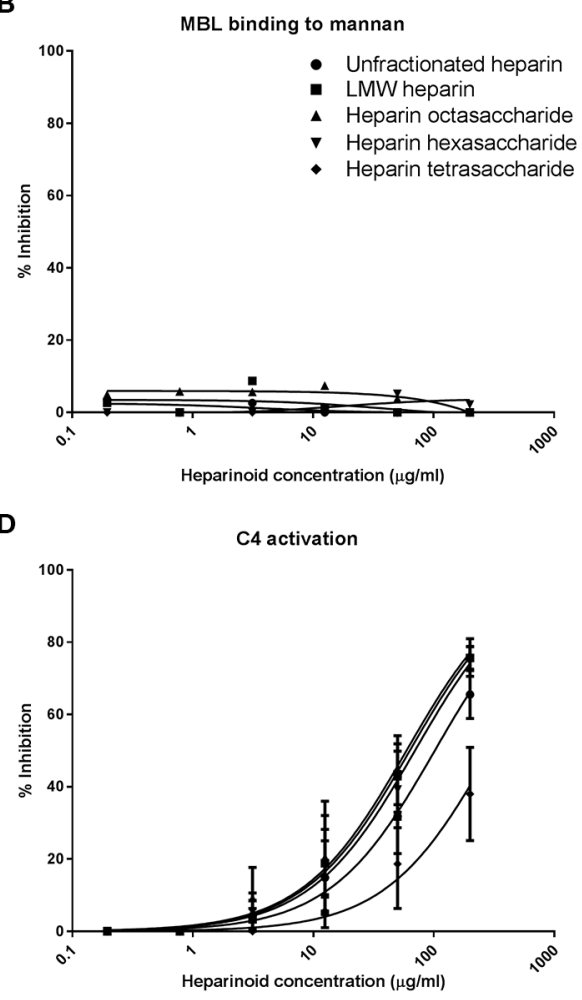

FIGURE 2 | Heparin-derived oligosaccharides inhibit the lectin pathway of complement via inhibition of C4d deposition. To determine which LP component is inhibited by heparin derivatives MBL and MASP inhibition were tested. Representative experiments show that MBL binds dose dependent to mannan (A). Heparin derivatives did not inhibit the binding of MBL to mannan (B). The selected heparin fragments did however show inhibition of the LP when the LP was initiated by ficolin-3 binding (C). Heparin fragments did also show an inhibitory effect in a C4d deposition assay, as a measure for MASP-2 activity (D). Data is expressed as a representative measurement (A-C) or as mean \pm SEM of three independent experiments (D).

of the MASPs. Since C4 is completely cleaved by MASP-2 and not by MASP-1, this is predominantly a MASP-2 assay (46). Interestingly, unfractionated heparin showed a relatively mild inhibitory potential (IC50: $102 \mu \mathrm{g} / \mathrm{ml}$ ) compared to the LMW-heparin enoxaparin, heparin oligosaccharide and heparin hexasaccharide (IC50: 63, 59, and $70 \mu \mathrm{g} / \mathrm{ml}$, respectively). The weakest inhibitory effect was shown by the heparin tetrasaccharide with an IC50 of $296 \mu \mathrm{g} / \mathrm{ml}$ (Figure 2D). This assay revealed that heparin oligosaccharides inhibit the LP at the level of the MASP enzymes, most likely MASP-2, in a dose dependent manner.

\section{Recombinant Human MASP-2 Shows Similar Binding Affinity for Short and Long HS GAGs}

To further strengthen the hypothesis that heparin/HS fragments predominantly inhibit MASP-2 we performed SPR experiments to evaluate the binding kinetics between catalytically active recombinant human MASP-2 fragment (CCP1-CCP2-SP) and different heparin/HS preparations. Figure $\mathbf{3 A}$ shows the sensorgram of recMASP-2 binding to an immobilized 15 $\mathrm{kDa}$ heparin fragment. Immobilized HS (porcine mucosa) and $6 \mathrm{kDa}$ LMW-heparin showed similar sensorgrams (Supplementary Figure 1). Interestingly the on and off rates were very fast indicating a transient interaction, which could indicate moderate affinity. In Figure 3B we show the fit of the experimental data for the binding of MASP-2 to $15 \mathrm{kDa}$ heparin upon representative steady-state analysis. Calculations of the affinity indeed show $\mathrm{Kd}$ values between 1.5 and $2.0 \mu \mathrm{M}$ for the heparins and HS tested (Figure 3C). The observation that MASP-2 showed similar affinity for the $6 \mathrm{kDa}$ heparin and the 15 $\mathrm{kDa}$ heparin and $\mathrm{HS}$ (usually between 12 and $20 \mathrm{kDa}$ ) indicates that MASP-2 recognizes a rather short and comparable motif on heparin/HS. Using the $15 \mathrm{kDa}$ heparin as a scaffold for MASP2 , competition assays were performed using unfractionated heparin, heparin-derived tetrasaccharide (identical composition as Org 32102, namely $\triangle$ HexA2S-GlCNS6S-IdoA2S-GlCNS6S) and chondroitin $\mathrm{A}, \mathrm{B}$, and $\mathrm{C}$ as competitors. Results show that the heparin-derived tetrasaccharide reduced the binding of MASP-2 to $15 \mathrm{kDa}$ heparin by $\pm 40 \%$. Although further experiments (including direct MASP-2/tetrasaccharide binding analysis) would be needed to ascertain that a tetrasaccharide is the actual minimal size required for MASP-2 binding, these data again suggest that MASP-2 is recognized by a short heparin/HS motif (Figure 3D). 
A

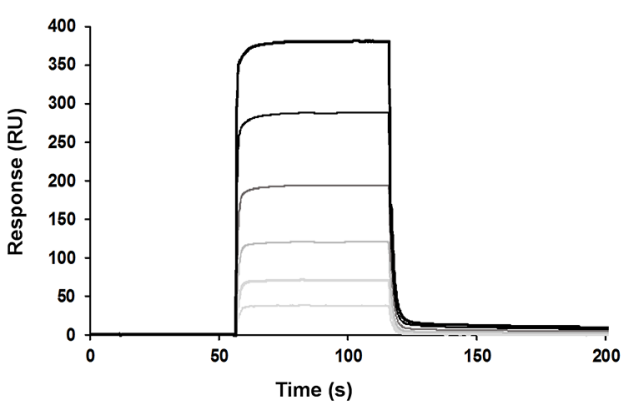

C

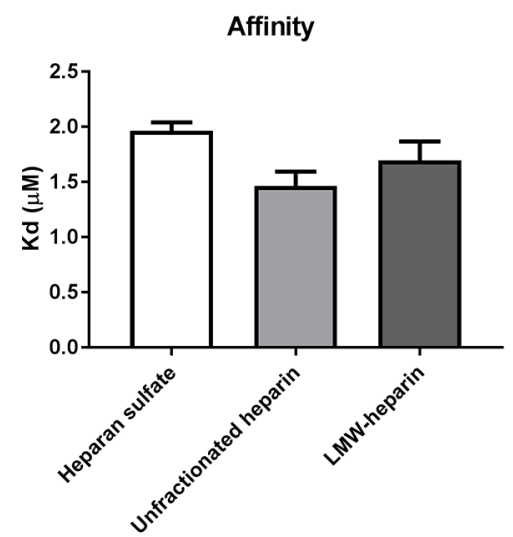

B

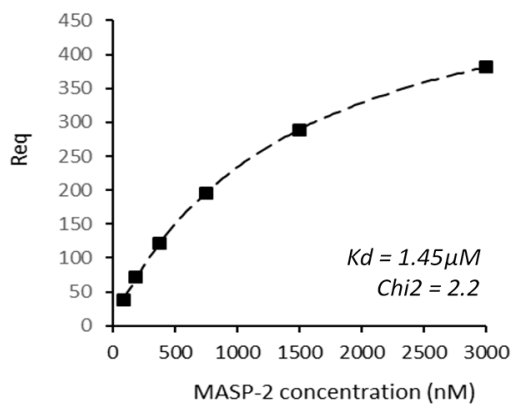

D

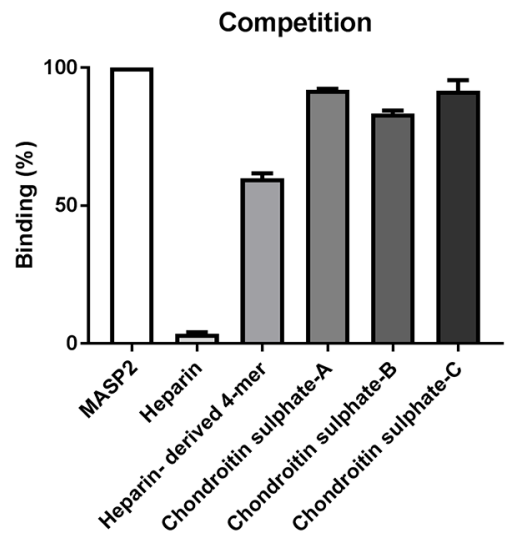

FIGURE 3 | The recombinant catalytic CCP1-CCP2-SP domain of MASP-2 shows equal affinity for heparan sulfate, small, and larger heparin preparations. (A) Surface plasmon resonance sensorgram of binding of a (from clear to dark lines) 0-3,000 nM concentration range of recombinant MASP-2 to $15 \mathrm{kDa}$ heparin, showing rapid on and off rates, indicating a transient interaction. Binding of MASP-2 to HS and 6 kDa heparin produced similar graphs (Supplementary Figure 1). (B) Representative steady-state analysis and fit of the experimental data for the binding of MASP-2 to $15 \mathrm{kDa}$ heparin. (C) Affinity calculations reveal micro molar affinity for MASP-2 binding to HS, $6 \mathrm{kDa}$, and $15 \mathrm{kDa}$ heparin. (D) Competition experiments using a tetrasaccharide (4-mer) heparin fragment results in a 40\% reduction of the binding of MASP-2 to immobilized $15 \mathrm{kDa}$ heparin, while chondroitin sulfates fail to inhibit the MASP-2/heparin interaction. Experiments were expressed as mean \pm SEM from three independent experiments.

\section{Sulfation Pattern Determines the MASP-2-Mediated Lectin Pathway Inhibitory Potential of Tetrasaccharides}

To determine the importance of sulfate group positioning within tetrasaccharides for the inhibition of the LP, various heparinderived tetrasaccharides obtained after limited heparinase I digestion as well as a set of synthetic tetrasaccharides were tested in the WieLISA and the C4 activation assay (37). As a reference the heparin tetrasaccharide Org 32102 (>90\% $\Delta$ UA2S-GlcNS6SIdoA2S-GlcNS6S as evaluated by NMR) and unfractionated heparin used in the experiments above were added. The tetrasaccharides were tested in both the WieLISA and MASP2-mediated C4 deposition assay. None of the tetrasaccharides affected the CP and the AP (data not shown). As shown before, results in the LP WieLISA and the MASP-mediated C4 deposition assay showed comparable results (Table 3 and Figure 4). Strongest inhibition in both assays was obtained by the fully hexasulfated $\Delta$ UA2S-GlcNS6S-IdoA2S-GlcNS6S (Org 32102) tetrasaccharide. The hexasulfated preparations with the $\Delta \mathrm{UA} 2 S$ at the non-reducing end (by heparinase I elimination reaction), like Org32102 and SAGAG peak 3, showed better inhibitory capacity compared to the synthetic variant, tetrasaccharide T40. Pentasulfated tetrasaccharides also showed inhibitory capacity in both assays, although to a lesser extent compared to the hexasulfated tetrasaccharide. Thus, SAGAG Peak 1 and 2 (having 5 sulfate groups) vs. SAGAG peak 3 (six sulfate groups), and synthetic tetrasaccharide T38 (having 5 sulfate groups) vs. T40 (having 6 sulfate groups). The data indicate that one of the 2-O or 6-O sulfates within the tetrasaccharide can be missed, leaving a pentasulfated $\mathrm{HS} /$ heparin tetrasaccharide being the minimal MASP-2 binding sequence in HS and heparin. Further reduction in sulfation to four or less sulfate groups/tetrasaccharide led to loss of all inhibitory potential in both the LP Wielisa and the MASP-2 C4 deposition assay (Table 3). Not tested in these experiments are the influence of 3-O sulfation and the eventual effect of GlcNAc or $\mathrm{GlcNH}_{3}^{+}$modifications. 
TABLE 3 | Inhibitory capacity of heparin-derived and synthetic tetrasaccharides in the Lectin Pathway WieLISA and MASP-mediated C4 deposition assay.

\begin{tabular}{|c|c|c|c|c|c|}
\hline Tetrasaccharide & Structure & Inhibition (\%) in WieLISA & Repeats & Inhibition (\%) in C4 assay & Repeats \\
\hline \multicolumn{6}{|c|}{ BY HEPARINASE I TREATMENT OF (LMW-) HEPARIN } \\
\hline Org 32102 & $\Delta$ UA2S-GIcNS6S-IdoA2S-GIcNS2S (90\%) & $76(2)$ & 3 & $61(6)$ & 3 \\
\hline \multirow[t]{5}{*}{ Ron G11237 } & $\Delta$ UA2S-GIcNS6S-IdoA2S-GIcNS2S (37\%) & 81 & 1 & ND & 1 \\
\hline & $\Delta$ UA2S-GlcNS6S-IdoA2S-AnS1,6an (18\%) & & & & \\
\hline & $\Delta$ UA2S-GlcNS6S-IdoA2S-ManNS6S (18\%) & & & & \\
\hline & $\Delta$ UA2S-GlcNS6S-GlcA-GlcNS3S6S (8\%) & & & & \\
\hline & DUA2S-GlcNS6S-GlcA-GlcNS6S (12\%) & & & & \\
\hline SAGAG Peak 1 & $\Delta U A 2 S-G l c N S 6 S+\Delta U A 2 S-G \mid c S^{c}$ & 46 & 1 & 55 & 1 \\
\hline SAGAG Peak 2 & $\Delta U A 2 S-G l c N S 6 S+\Delta U A-G l c N S 6 S^{c}$ & 28 & 1 & 47 & 1 \\
\hline SAGAG Peak 3 & $\Delta \mathrm{UA2S}-\mathrm{GlcNS6S}+\Delta \mathrm{UA2S}-\mathrm{GlcNS6S^{c }}$ & 68 & 1 & 66 & 1 \\
\hline \multicolumn{6}{|c|}{ SYNTHETIC TETRASACCHARIDES } \\
\hline $\mathrm{T} 7$ & IdoA-GlcNAc6S-IdoA-GlcNAc6S- $\left(\mathrm{CH}_{2}\right)_{5} \mathrm{NH}_{2}$ & 0 & 1 & $0(0)$ & 3 \\
\hline T11 & IdoA-GlcNAc6S-IdoA2S-GlcNAc6S- $\left(\mathrm{CH}_{2}\right)_{5} \mathrm{NH}_{2}$ & 0 & 1 & $0(0)$ & 3 \\
\hline T13 & IdoA-GlcNS6S-IdoA-GlcNS6S- $\left(\mathrm{CH}_{2}\right)_{5} \mathrm{NH}_{2}$ & 0 & 1 & $0(0)$ & 3 \\
\hline T38 & IdoA-GlcNS6S-IdoA2S-GIcNS6S- $\left(\mathrm{CH}_{2}\right)_{5} \mathrm{NH}_{2}$ & 24 & 1 & $10(10)$ & 3 \\
\hline T39 & IdoA2S-GlcNAc6S-IdoA2S-GlcNAc6S- $\left(\mathrm{CH}_{2}\right)_{5} \mathrm{NH}_{2}$ & 0 & 1 & $0(0)$ & 3 \\
\hline T40 & IdoA2S-GlcNS6S-IdoA2S-GlcNS6S- $\left(\mathrm{CH}_{2}\right)_{5} \mathrm{NH}_{2}$ & 30 & 1 & $16(3)$ & 3 \\
\hline
\end{tabular}

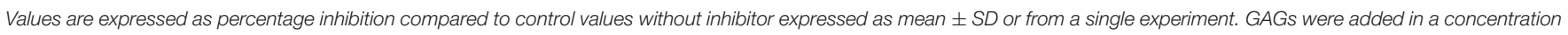
of $100 \mathrm{ug} / \mathrm{ml}$ in the LP and C4 assay.

${ }^{a}$ Composition and purity by NMR spectroscopy.

${ }^{b}$ Composition by NMR spectroscopy.

${ }^{c}$ Composition after digestion of the tetrasaccharide with a cocktail of heparinase I, II, and III followed by RPIP-HPLC disaccharide identification.

\section{MBL-MASP Complex Binds to Immobilized Heparin and Tissue Heparan Sulfate}

We have shown in this study that specific domains in heparins and HS can inhibit the LP of complement by inhibiting the enzymatic activity of the MASP2 enzyme. To test whether the MASP-2 in serum indeed binds to solid phase heparin/HS, we incubated serum in heparin-albumin coated wells and measured whether MASP-2 was bound to the immobilized heparin. Results showed binding of serum-derived MASP-2 to immobilized heparin in a dose dependent fashion (Figure 5A), indicating that serum MASP-2 could bind to HS/heparin on cells and tissues. RecMASP-2 showed binding to heparin albumin as well (Figure 5B).

We thus hypothesized that HS in extracellular matrices and cell membranes could function as docking platforms for MASP-2 in vivo. To test this hypothesis we double stained human donor kidneys for MASP-2 and basement membrane HS proteoglycan agrin, which is localized in renal glomerular, tubular and vascular basement membranes. We also performed a double staining for MASP-2 and HS (mAb 10E4). Both double stainings revealed MASP-2 to be present in a subset of tubular basement membranes, partly colocalizing with agrin and HS (Figures 5C-H, 6A-C). The fact that not all basement membranes showed MASP- 2 positivity might be because MASP2 is just very locally produced and stored in the underneath basement membrane, and/or that just a small subset of renal basement membranes displayed HS with a MASP-2 binding motif.

However, from these data it is not clear whether MASP-2 is enzymatically active or not when co-localized to basement membrane HSPGs. Therefore, we performed a triple staining for HS using mAb 10E4, MASP-2 and C3b, the cleaved product of C3, also called active C3. Although the 10E4 and MASP-2 epitopes of HS are different from each other, in Figures 6D-G it can be appreciated that MASP-2 and HS showed partial co localization in some basement membranes of vascular structures and in Bowman's capsule. Cleaved C3 within the glomerulus did not colocalize with MASP-2 and/or HS. It can also be seen that cleaved C3 occasionally showed co localization with MASP-2, but not when MASP-2 is co localized with 10E4/HS. Within the limitations of this approach, we speculate that MASP-2 bound to tissue HS is not able to activate the LP and thus no C3b can be formed i.e., MASP-2 might be enzymatically inhibited by the binding to HSPGs.

\section{DISCUSSION}

The interaction of GAGs (especially heparin-related GAGs) with complement has been known for some decades, but it has never been tested on a larger scale whether GAGs and derivatives thereof could be specific inhibitors of either of the complement pathways. In this study we tested a library of GAG-related polysaccharides for their complement inhibitory capacity and showed that the LP of complement can be specifically blocked by some HS and heparin- and HS-derived oligosaccharides. Decreasing the disaccharide length of the HS and heparin oligosaccharides resulted in more specificity for the LP and loss of CP and AP inhibitory activity. Since the LP shares the C3 convertase C4b2a with the CP, LP-specific GAGs must inhibit either the MASP enzymes or the pattern recognition molecules 


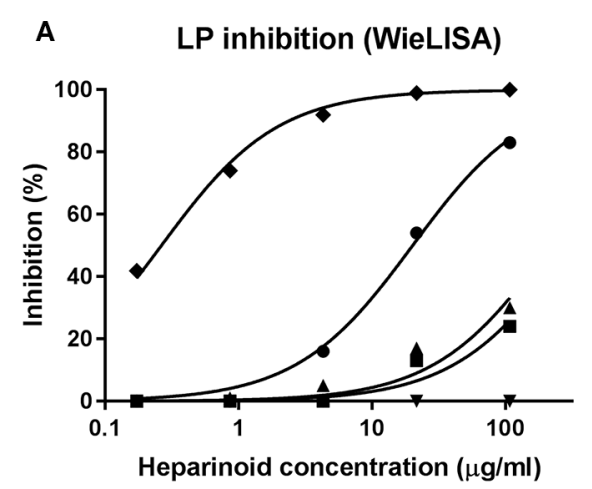

C

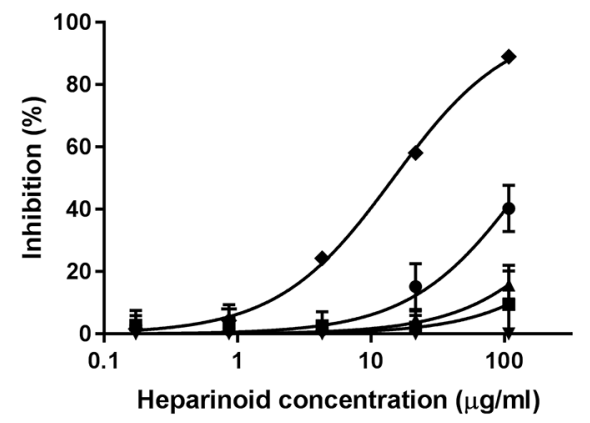

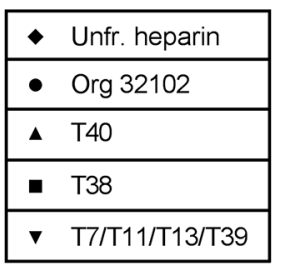

B

\section{LP inhibition (WieLISA)}

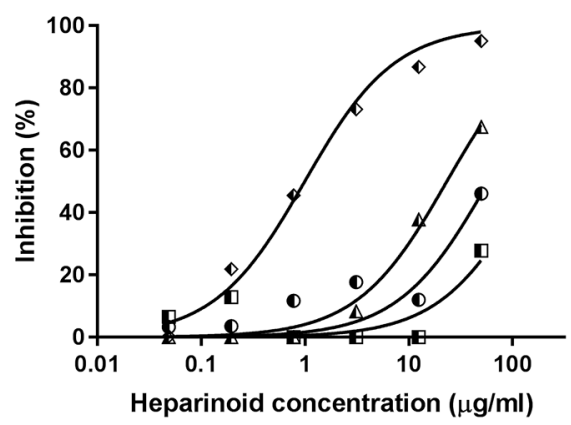

D

C4 activation

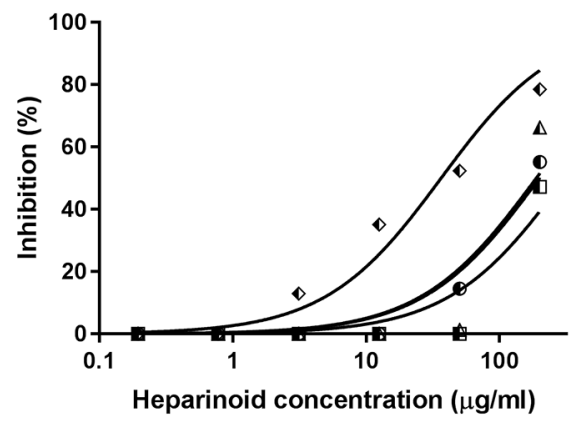

FIGURE 4 | Inhibition of the lectin pathway by tetrasaccharides requires at least pentasulfation. Using synthetic tetrasaccharides (A,C) and purified tetrasaccharides (B,D), we determined that LP inhibition requires at least a pentasulfated tetrasaccharide. All penta- and hexa-sulfated oligosaccharides showed inhibition in both the LP WieLISA and the C4 activation test. Removing both IdoA2S sulfate groups or both $\mathrm{N}$-sulfate groups resulted in vanishing of the inhibitory potential. Displacement of an iduronic acid by an unsaturated uronic or hexuronic acid results in an improvement of inhibitory potential in both assays. For data on the sulfation degree of the tetrasaccharides see Table 3. As a reference the non-synthetic heparin tetrasaccharide 32102 and unfractionated heparin, used in the former experiments, was added to experiment $(\mathbf{A}, \mathbf{C})$. Data is expressed as representative measurement $(\mathbf{A}, \mathbf{B}, \mathbf{D})$ or as the mean \pm SEM of three independent experiments $(\mathbf{C})$.

of the LP. Our results show that heparin fragments inhibited the cleaving of $\mathrm{C} 4$, but did not affect the binding of MBL to mannan, suggesting a blocking effect of GAGs on either MASP-1 or MASP2 activity. This conclusion is further strengthened by the finding that the tested GAGs are also able to inhibit the LP initiated by ficolin-3 as a pattern recognition molecule. We could also show binding of serum-derived MASP-2 as well as recMASP-2 to immobilized heparin and demonstrated the binding affinity of recombinant MASP-2 to heparins and HS. Moreover, partial colocalization was seen of MASP-2 and agrin/10E4 HS epitope in some basement membrane of tubuli and vessels in human kidney, while activated C3 did not co-localize. These data might suggest that HS in situ might function as a docking platform for MASP-2 rendering the enzyme inactive, thereby eventually protecting the tissue from LP complement activation.
GAG length, disaccharide backbone composition, and sulfation pattern form the main determinants for protein binding properties of GAGs in general and this study shows that this holds true for complement components as well. Although the heparin/HS (oligosaccharides) were tested in a fixed $\mu \mathrm{g} / \mathrm{ml}$ concentration, increasing the difficulty to directly compare small and large fractions, it can be appreciated that in larger GAGs, the complement inhibitory capacity is predominantly determined by sulfation degree and less by disaccharide composition and linkages. GAGs carrying a different backbone compared to heparin like O-sulfated E. coli K5 polysaccharide, and GAG-like polysaccharides fucoidan and dextran sulfate show equally strong inhibition of all complement pathways, stressing the role of GAG sulfation in complement inhibition. It can be appreciated from Table 2 that in most cases the inhibitory 

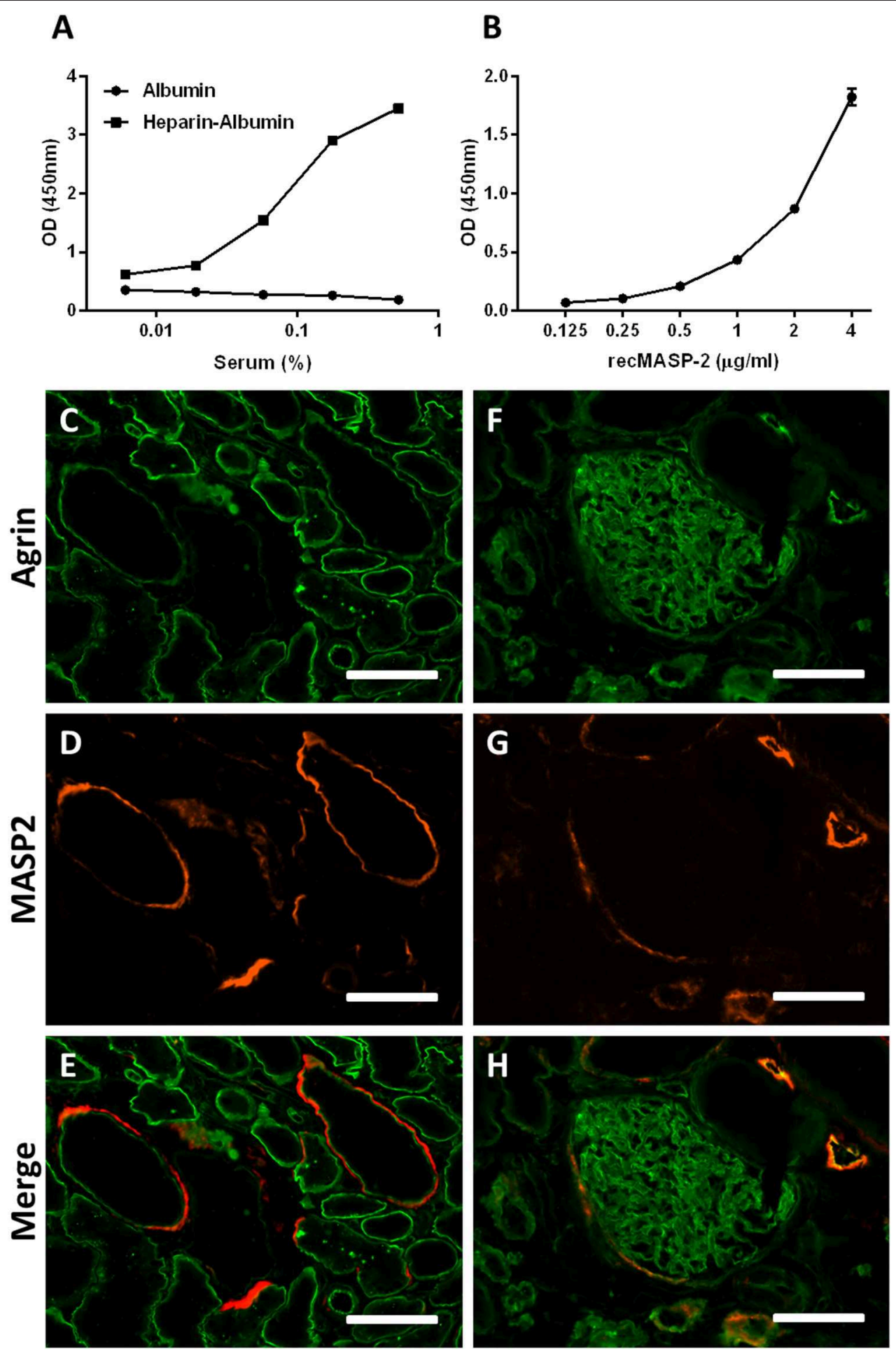

Tubuli

\section{Glomerulus}

FIGURE 5 | HSPG's might serve as a docking platform for the MBL/MASP complex. (A) Incubation of serum on a heparin-albumin coated plate results in binding of MASP-2 to the heparin. (B) recMASP-2 binds to heparin-albumin coated on a plate. (C-H) Confocal immunofluorescence double staining of human donor kidneys for MASP-2 (red) and agrin (green) showed partial co localization in the basement membranes of some tubuli, within Bowman's capsule and some blood/lymph vessel basement membranes. Data is expressed as representative measurement (A) or as mean \pm SEM of two independent experiments (B). Scale bars indicate $50 \mu \mathrm{m}$. 


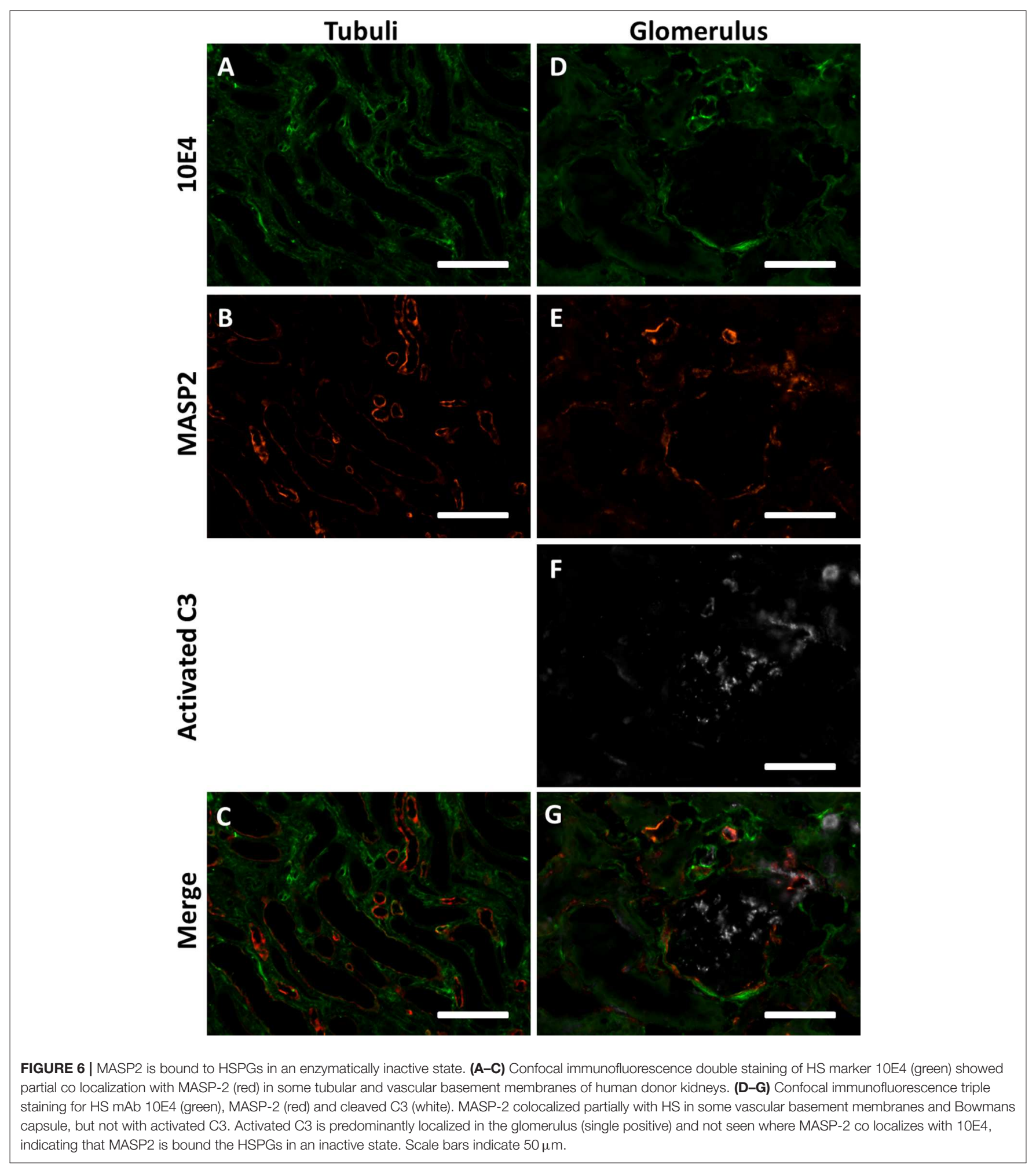

capacity of GAGs is comparable for the CP and AP. This might suggest that the $\mathrm{CP}$ and AP share an inhibitory GAG binding protein and that the GAG binding site in the LP is unique within the complement system. The known interactions between GAGs and the complement system have been summarized by us before (47) and we have more extensively investigated the interaction between GAGs and properdin (12). Interestingly, the GAGs showing the highest affinity for properdin in the study conducted 
earlier also show the strongest CP and AP inhibition (12). This might indicate a role for the GAG/properdin interaction in our current results. Properdin is a stimulator of the AP, by stabilizing the $\mathrm{C} 3$ convertase, but can have the same effect in the $\mathrm{CP}$ since cleaving of $\mathrm{C} 3$ by the $\mathrm{C} 3$ convertase $\mathrm{C} 4 \mathrm{bC} 2 \mathrm{a}$ can be followed by $\mathrm{AP}$ activation. Whether the inhibition by GAGs of the CP and AP is indeed properdin mediated or not should be studied further, since interactions between GAGs on one hand and AP activator properdin, AP inhibitor factor $\mathrm{H}$ (13) and CP inhibitors like C1-inhibitor on the other hand have been described Caughman et al. (48).

This study showed that smaller heparin and HS depolymerization products lose their ability to inhibit the $\mathrm{CP}$ and AP and become specific inhibitors for the LP. HS oligosaccharides start to be LP specific from dp12 and heparin from dp6. Our surface plasmon resonance experiments indicated that MASP-2 recognizes a small GAG epitope. Of note, heparin is significantly more sulfated than HS $(\sim 2.5$ and $\sim 0.85$ sulfates per disaccharide, respectively). Furthermore, HS oligosaccharides were generated using heparinase III, which specificity for (GlcNAc $\pm 6 \mathrm{~S}-\mathrm{GlcA}$ ) disaccharides will produce fragments with low/non-sulfated saccharide extremities. We therefore assume that larger (dp6-dp12) HS oligosaccharides may contain just one MASP2 binding site connected to low sulfated, biologically inactive HS stretches. Whereas, larger (dp6-dp12) heparin oligo's, due to high sulfation, also start to recognize Clinh, ATIII and other members of the complement serine proteases and therefore start to inhibit the CP and AP besides the LP. The smallest component capable of inhibiting the LP is a tetrasaccharide, which needs to have at least pentasulfation to show inhibitory function. This is interesting because other GAG/protein interactions e.g., heparin and ATIII have shown to require larger high sulfation stretches. Therefore, the interaction of a tetrasaccharide with MASP enzymes holds clinical potential. Interestingly, replacing the iduronic acid at the non-reducing end of the tetrasaccharide by unsaturated $\Delta \mathrm{HexA}$ could increase the inhibitory potential, suggesting the importance of the backbone structure. Alternatively, the reducing terminal- $\left(\mathrm{CH}_{2}\right)_{5} \mathrm{NH}_{2}$ derivatization of the synthetic tetrasaccharides might interfere the interaction with MASP-2 to some extent.

We showed in this study that the inhibitory effect of GAGs on the lectin route of complement is not pattern recognition molecule related, but rather MASP related. As elegantly shown by Héja and colleagues, MASP-2 is solely responsible for the activation of C4 and both MASP-1 and 2 are responsible for the activation of C2 (46). This study shows that GAGs inhibit the C4 activation and that GAGs show interaction with the catalytic domain of MASP-2. Therefore, we can conclude that GAGs interact with MASP-2 and inhibit the enzymatic activity. Literature has demonstrated that MASP-2, but not MASP-1, has a highly positively charged exosite located in the serine protease domain, which differs from de described exosite in $\mathrm{C} 1 \mathrm{~s}$ $(49,50)$. Under physiological conditions, this positively charged exosite is believed to be the binding site of $\mathrm{C} 4$. C4 carries a negatively charged cluster consisting of three tyrosine-sulfate residues, interaction of these tyrosine-sulfate residues with the positively charged exosite on MASP-2 and C1s is crucial for cleaving, and therefore activity, of $\mathrm{C} 4(51,52)$. Since heparin, and HS to a lesser extent, is negatively charged due to their sulfate groups, we propose that specific LP inhibition by HS and heparin oligosaccharides occurs via binding to this positively charged exosite. In Figure 7, we aligned the amino acid sequence of the serine protease domains of MASP-2, MASP-1, and C1s. In green we indicated the positively charged arginine and lysine residues in MASP-2 involved in the recognition of the tyrosine-sulfate residues in C4 (K450, K503, R578, and R583). In red we showed them in C1s (K575, R576, R581, and K583). As can be seen, such an exosite is lacking in MASP-1. Moreover, MASP-1 is not cleaving $\mathrm{C} 4$, just $\mathrm{C} 2$. Moreover, because we found the inhibition of the C4 deposition assay we studied MASP-2 and not MASP1. Therefore, these data strongly suggest that the heparin oligo's block the interaction of MASP-2/C4 interaction at the interface of the MASP-2 exosite with the tyrosin-sulfate residues of $\mathrm{C} 4$, and thereby prevents cleavage of $\mathrm{C} 4$.

It has been described that protease inhibitors like Clinh, ATIII, and tissue factor pathway inhibitor can form complexes with the MASP proteases $(15,54)$ and could therefore influence our results by heparin mediated activation of these serine protease inhibitors followed by inactivation of the MASP enzymes by proteolytic cleavage. We deem it however unlikely since ATIII needs minimally a $3-\mathrm{O}$ sulfated pentasaccharide which is not present in the synthetic tetrasaccharides tested (25). Clinh and TFPI are also not very likely involved, since according to literature both protease inhibitors show an increased protease activity at low heparin concentrations and blocking of the protease activity at high heparin concentrations $(15,55)$. We never observed such kinetics using the WIELISA or C4 deposition assays. Besides, the $1 \mathrm{M} \mathrm{NaCl}$ conditions in the $\mathrm{C} 4$ deposition assay the MASPs will not react with the serpins.

This study shows that the serum-derived MASP-2 can bind to heparin and HS GAGs and inhibit the LP of complement. These findings suggest that HS proteoglycans in vivo can act as a docking station for MASP-2 and preclude LP activity. In support to this, we found some co-localization of MASP-2 and HS proteoglycan agrin and HS in Bowman's capsule and the basement membranes of some tubuli and vessels. We were not able to show cleaved C3 to co-localize with HS and MASP2. To test HS-dependent MASP-2 binding renal sections were pretreated with heparitinase. Effective enzymatic cleavage of HS was shown by induced staining using anti- $\Delta \mathrm{HS}$ stub $\mathrm{mAb}$ 3G10, which after heparitinase became brilliant positive in all extracellular matrices of the kidney. Nevertheless, the intensity of the MASP-2 staining did not diminish after heparitinase (data not shown). This could mean that MASP-2 is not physically bound with HS. However, we cannot exclude that its binding to HS precludes the heparitinase enzyme to cleave HS. Another explanation could be that the MASP-2 in renal tissue is part of a larger complement complex, including MASP-1, MBL, and eventually other complement factors (C2 and C4 eg). These other complement factors also might interact with renal determinants, other than HS. This means that digestion of HS will not reduce MASP-2 presence. All in all, such an enzymatic approach is not conclusive. Although our immunofluorescence data is far from robust proof, this might suggest that MASP-2 is stored inactively 


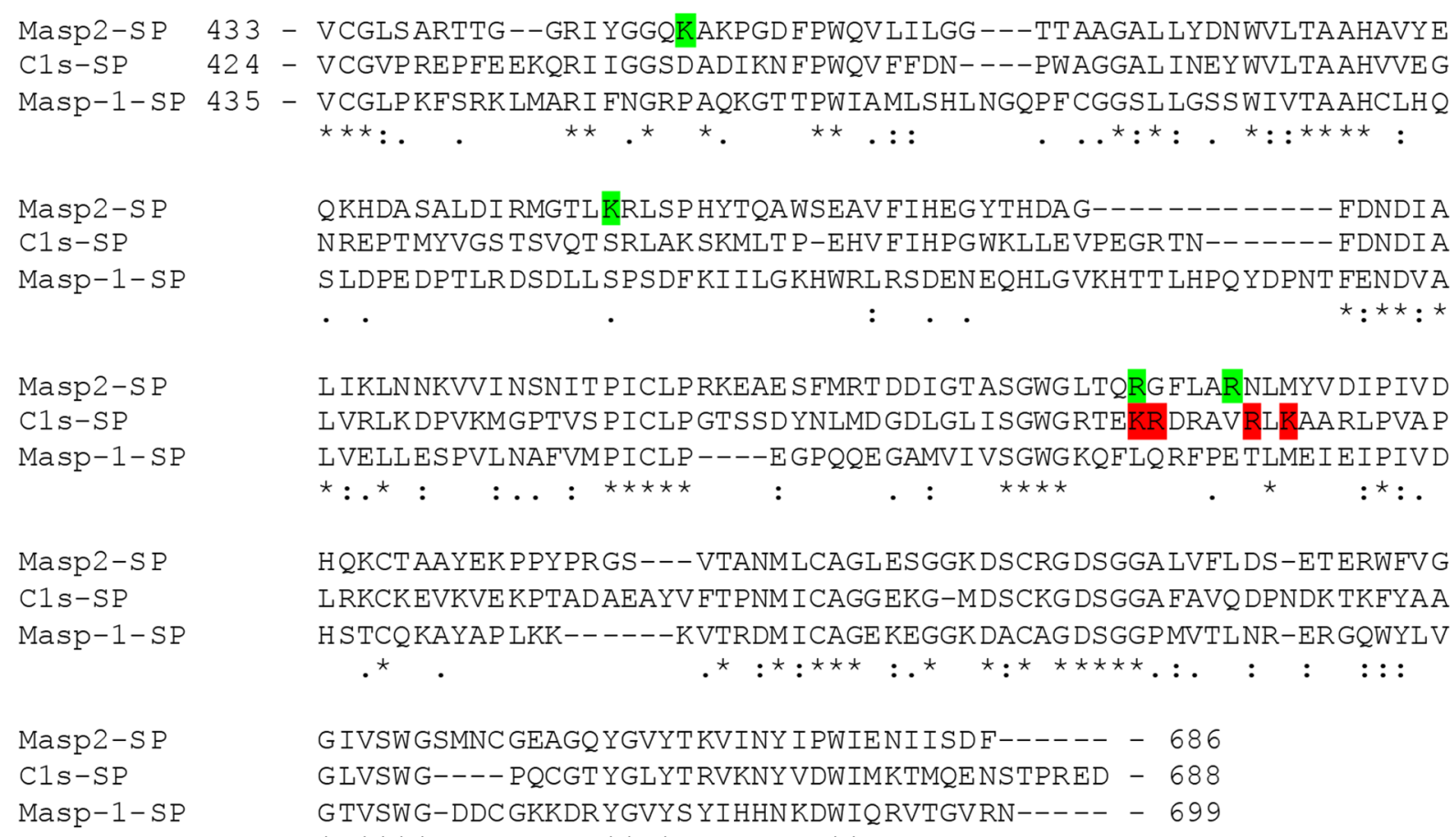

FIGURE 7 | Amino acid sequence alignment of the serine protease domains of MASP-2, C1s and MASP-1. Sequence alignment was done by Clustal W (https:// www.genome.jp/tools-bin/clustalw) as described by Thompson et al. (53). Amino acid sequences were obtained in Uniprot (https://www.uniprot.org/). Indicated in green are the positively charged arginine and lysine residues in MASP-2 involved in the recognition of the tyrosine-sulfate residues in C4 (K450, K503, R578, and R583). In red these residues are shown in C1s (K575, R576, R581, and K583). As can be seen, the positively charged exocites of MASP-2 and C1s clearly differs from each other, while such a positively charged exosite is lacking in MASP-1.

in the basement membrane and we speculate that it might be released upon the right stimuli e.g., by the action of heparanase or oxidative stress.

We show in this study that HS and small heparin fragments are specific LP inhibitors, although the interaction is in the micromolar affinity range resulting in rather high IC50 values. To date there is no specific LP inhibitor clinically available, although a phase II clinical trial in renal patients using humanized anti-MASP-2 mAb (OMS721 by OMEROS, Saettle WA, USA) was recently closed with promising results in patients suffering from IgA nephropathy and lupus nephritis. Furthermore, an interesting study was recently published introducing a modified TFPI 1 domain as a potential MASP2 inhibitor (56). Current treatment option for LP mediated conditions would be C5 inhibitor eculizumab. However, eculizumab is very expensive and inhibits all three complement pathways. The use of polysaccharide-based therapeutics has the advantages: production can be cheap and easy, and polysaccharides are not susceptible to proteolytic enzymes, making them less vulnerable to degradation. Moreover, decades of experience has been obtained with heparin based therapeutics in the field of anti-coagulation. Therefore, we believe that polysaccharide based specific LP inhibitors have good potential for further development. The comparison of four different $\beta$-elimination heparin-derived tetrasaccharide fragments and synthetic tetrasaccharides revealed the best LP/MASP inhibition by the fully hexasulfated $\Delta \mathrm{HexA}, 2 \mathrm{~S}-\mathrm{GlcNS}, 6 \mathrm{~S}-\Delta \mathrm{HexA}, 2 \mathrm{~S}-$ GlcNS, 6S structure. This structure could be a promising starting molecule to develop related small glycan-based LP/MASP inhibitors with higher affinity.

\section{DATA AVAILABILITY STATEMENT}

All datasets generated for this study are included in the article/Supplementary Material.

\section{AUTHOR CONTRIBUTIONS}

DT, MD, CS, MS, SB, and JB contributed to the conception and design of the study. DT, FP, WD, AM-A, and RV conducted the experiments. AN, PC, G-JB, and PG delivered crucial reagents. DT wrote the first draft of the manuscript. All authors contributed to manuscript revision, read, and approved the submitted version.

\section{FUNDING}

The research was funded by the graduate school of Medical Sciences of the University Medical Center of Groningen in 
the form of a Ph.D. stipend to DT and by the Dutch Kidney Foundation (grants IP 12.90 and Consortium grant COMBAT \#130CA27. This work used the SPR platform of the Grenoble Instruct centre (ISBG; UMS 3518 CNRS-CEAUJF-EMBL) with support from FRISBI (ANR-10-INSB-0502) and GRAL (ANR-10-LABX-49-01) within the Grenoble Partnership for Structural Biology (PSB). This work was also supported by the CNRS and the GDR GAG (GDR 3739), the Investissements d'avenir program Glyco@Alps (ANR-15-IDEX02), and by a Agence Nationale de la Recherche grant (ANR17-CE11-0040). The study was supported by the Hungarian National Research, Development and Innovation Office OTKA grant K119374.

\section{ACKNOWLEDGMENTS}

We thank Justyna Dobruchowska for performing the structural analysis of the Org 32102 tetrasaccharide and Evelyne Gout for technical help with the SPR experiments. We are grateful to Júlia Balczer for the technical assistance. This study was performed on behalf of the COMBAT Consortium. This is an interuniversity collaboration in the Netherlands that is formed to study basic mechanisms, assay development, and therapeutic translation of

\section{REFERENCES}

1. Thurman JM, Nester CM. All things complement. Clin J Am Soc Nephrol. (2016) 11:1856-66. doi: 10.2215/CJN.01710216

2. Leffler J, Bengtsson AA, Blom AM. The complement system in systemic lupus erythematosus: an update. Ann Rheum Dis. (2014) 73:16016. doi: 10.1136/annrheumdis-2014-205287

3. Hovind P, Hansen TK, Tarnow L, Thiel S, Steffensen R, Flyvbjerg A, et al. Mannose-binding lectin as a predictor of microalbuminuria in type 1 diabetes: an inception cohort study. Diabetes. (2005) 54:15237. doi: 10.2337/diabetes.54.5.1523

4. Berger SP, Roos A, Mallat MJ, Fujita T, de Fijter JW, Daha MR. Association between mannose-binding lectin levels and graft survival in kidney transplantation. Am J Transplant. (2005) 5:1361-6. doi: 10.1111/j.1600-6143.2005.00841.x

5. Roos A, Rastaldi MP, Calvaresi N, Oortwijn BD, Schlagwein N, van GijlswijkJanssen DJ, et al. Glomerular activation of the lectin pathway of complement in IgA nephropathy is associated with more severe renal disease. J Am Soc Nephrol. (2006) 17:1724-34. doi: 10.1681/ASN.2005090923

6. Poppelaars F, Faria B, Gaya da Costa M, Franssen CFM, van Son WJ, Berger SP, et al. The complement system in dialysis: a forgotten story? Front Immunol. (2018) 9:71. doi: 10.3389/fimmu.2018.00071

7. Moller-Kristensen M, Wang W, Ruseva M, Thiel S, Nielsen S, Takahashi K, et al. Mannan-binding lectin recognizes structures on ischaemic reperfused mouse kidneys and is implicated in tissue injury. Scand J Immunol. (2005) 61:426-434. doi: 10.1111/j.1365-3083.2005.01591.x

8. Farrar CA, Tran D, Li K, Wu W, Peng Q, Schwaeble W, et al. Collectin-11 detects stress-induced L-fucose pattern to trigger renal epithelial injury. J Clin Invest. (2016) 126:1911-25. doi: 10.1172/JCI83000

9. Gaarkeuken H, Siezenga MA, Zuidwijk K, van Kooten C, Rabelink TJ, Daha MR, et al. Complement activation by tubular cells is mediated by properdin binding. Am J Physiol Renal Physiol. (2008) 295:F1397403. doi: 10.1152/ajprenal.90313.2008

10. Hawfield A, Iskandar SS, Smith RJ. Alternative pathway dysfunction in kidney disease: a case report and review of dense deposit disease and C3 glomerulopathy. Am J Kidney Dis. (2013) 61:828-31. doi: 10.1053/j.ajkd.2012.11.045 complement-mediated renal diseases. Principal investigators are (in alphabetical order): SB (Department of Internal MedicineNephrology, University Medical Center Groningen, Groningen, Netherlands), JB (Department of Internal Medicine-Nephrology, University Medical Center Groningen, Groningen, Netherlands), P. Gros (Department of Chemistry, Utrecht University, Utrecht, Netherlands), L.P. van den Heuvel (Department of Pediatric Nephrology, Radboud University Medical Center, Nijmegen, Netherlands), N. van de Kar (Department of Pediatric Nephrology, Radboud University Medical Center, Nijmegen, Netherlands), C. van Kooten (Department of Internal Medicine-Nephrology, Leiden University Medical Center, Leiden, Netherlands), MS (Department of Internal Medicine-Nephrology, University Medical Center Groningen, Groningen, Netherlands), A. de Vries (Department of Internal Medicine-Nephrology, Leiden University Medical Center, Leiden, Netherlands).

\section{SUPPLEMENTARY MATERIAL}

The Supplementary Material for this article can be found online at: https://www.frontiersin.org/articles/10.3389/fimmu. 2020.00732/full\#supplementary-material

11. Sethi S, Fervenza FC. Pathology of renal diseases associated with dysfunction of the alternative pathway of complement: C3 glomerulopathy and atypical hemolytic uremic syndrome (aHUS). Semin Thromb Hemost. (2014) 40:41621. doi: 10.1055/s-0034-1375701

12. Zaferani A, Vives RR, van der Pol P, Hakvoort JJ, Navis GJ, van Goor H, et al. Identification of tubular heparan sulfate as a docking platform for the alternative complement component properdin in proteinuric renal disease. $J$ Biol Chem. (2011) 286:5359-67. doi: 10.1074/jbc.M110.167825

13. Zaferani A, Vives RR, van der Pol P, Navis GJ, Daha MR, van Kooten $\mathrm{C}$, et al. Factor $\mathrm{h}$ and properdin recognize different epitopes on renal tubular epithelial heparan sulfate. J Biol Chem. (2012) 287:3147181. doi: 10.1074/jbc.M112.380386

14. Weiler JM, Edens RE, Linhardt RJ, Kapelanski DP. Heparin and modified heparin inhibit complement activation in vivo. J Immunol. (1992) 148:321015.

15. Parej K, Dobo J, Zavodszky P, Gal P. The control of the complement lectin pathway activation revisited: both $\mathrm{C} 1$-inhibitor and antithrombin are likely physiological inhibitors, while alpha2-macroglobulin is not. Mol Immunol. (2013) 54:415-22. doi: 10.1016/j.molimm.2013.01.009

16. Rent R, Myhrman R, Fiedel BA, Gewurz H. Potentiation of C1-esterase inhibitor activity by heparin. Clin Exp Immunol. (1976) 23:264-71.

17. Loos M, Bitter-Suermann D. Mode of interaction of different polyanions with the first $(\mathrm{C} 1, \mathrm{C} 1)$ the second $(\mathrm{C} 2)$ and the fourth $(\mathrm{C} 4)$ component of complement. IV. Activation of $\mathrm{C} 1$ in serum by polyanions. Immunology. (1976) 31:931-4. doi: 10.1016/0161-5890(76)90026-2

18. Poppelaars F, Damman J, de Vrij EL, Burgerhof JG, Saye J, Daha MR, et al. New insight into the effects of heparinoids on complement inhibition by C1-inhibitor. Clin Exp Immunol. (2016) 184:378-88. doi: 10.1111/cei.12777

19. Talsma DT, Daha MR, van den Born J. The bittersweet taste of tubulo-interstitial glycans. Nephrol Dial Transplant. (2017) 32:611-19. doi: 10.1093/ndt/gfw371

20. Sarrazin S, Lamanna WC, Esko JD. Heparan sulfate proteoglycans. Cold Spring Harb Perspect Biol. (2011) 3:a004952. doi: 10.1101/cshperspect.a0 04952

21. Vlodavsky I, Beckhove P, Lerner I, Pisano C, Meirovitz A, Ilan N, et al. Significance of heparanase in cancer and inflammation. Cancer Microenviron. (2012) 5:115-32. doi: 10.1007/s12307-011-0082-7 
22. El Masri R, Seffouh A, Lortat-Jacob H, Vives RR. The "in and out" of glucosamine 6-O-sulfation: the 6th sense of heparan sulfate. Glycoconj J. (2017) 34:285-98. doi: 10.1007/s10719-016-9736-5

23. Celie JW, Reijmers RM, Slot EM, Beelen RH, Spaargaren M, Ter Wee PM, et al. Tubulointerstitial heparan sulfate proteoglycan changes in human renal diseases correlate with leukocyte influx and proteinuria. Am J Physiol Renal Physiol. (2008) 294:F253-63. doi: 10.1152/ajprenal.00429.2007

24. Katta K, Boersema M, Adepu S, Rienstra H, Celie JW, Mencke R, et al. Renal heparan sulfate proteoglycans modulate fibroblast growth factor 2 signaling in experimental chronic transplant dysfunction. Am J Pathol. (2013) 183:1571-84. doi: 10.1016/j.ajpath.2013.07.030

25. Casu B, Lindahl U. Structure and biological interactions of heparin and heparan sulfate. Adv Carbohydr Chem Biochem. (2001) 57:159206. doi: 10.1016/S0065-2318(01)57017-1

26. Vann WF, Schmidt MA, Jann B, Jann K. The structure of the capsular polysaccharide (K5 antigen) of urinary-tract-infective Escherichia coli 010:K5:H4. A polymer similar to desulfo-heparin. Eur J Biochem. (1981) 116:359-64. doi: 10.1111/j.1432-1033.1981.tb05343.x

27. Maccarana M, Sakura Y, Tawada A, Yoshida K, Lindahl U. Domain structure of heparan sulfates from bovine organs. J Biol Chem. (1996) 271:1780410. doi: $10.1074 /$ jbc. 271.30 .17804

28. Iverius PH. Coupling of glycosaminoglycans to agarose beads (sepharose 4B). Biochem J. (1971) 124:677-83. doi: 10.1042/bj1240677

29. Rej RN, Ludwig-Baxter KG, Perlin AS. Sulfation of some chemically-modified heparins. Formation of a 3-sulfate analog of heparin. Carbohydr Res. (1991) 210:299-310. doi: 10.1016/0008-6215(91)80130-F

30. Vives RR, Sadir R, Imberty A, Rencurosi A, Lortat-Jacob H. A kinetics and modeling study of RANTES(9-68) binding to heparin reveals a mechanism of cooperative oligomerization. Biochemistry. (2002) 41:1477989. doi: $10.1021 /$ bi026459i

31. Pye DA, Vives RR, Turnbull JE, Hyde P, Gallagher JT. Heparan sulfate oligosaccharides require 6-O-sulfation for promotion of basic fibroblast growth factor mitogenic activity. J Biol Chem. (1998) 273:2293642. doi: $10.1074 /$ jbc. 273.36 .22936

32. Henriet E, Jager S, Tran C, Bastien P, Michelet JF, Minondo AM, et al. A jasmonic acid derivative improves skin healing and induces changes in proteoglycan expression and glycosaminoglycan structure. Biochim Biophys Acta Gen Subj. (2017) 1861:2250-60. doi: 10.1016/j.bbagen.2017.06.006

33. Seffouh A, Milz F, Przybylski C, Laguri C, Oosterhof A, Bourcier S, et al. HSulf sulfatases catalyze processive and oriented 6-O-desulfation of heparan sulfate that differentially regulates fibroblast growth factor activity. FASEB J. (2013) 27:2431-9. doi: 10.1096/fj.12-226373

34. Chabrol E, Nurisso A, Daina A, Vassal-Stermann E, Thepaut M, Girard E, et al. Glycosaminoglycans are interactants of langerin: comparison with gp120 highlights an unexpected calcium-independent binding mode. PLoS ONE. (2012) 7:e50722. doi: 10.1371/journal.pone.0050722

35. Alekseeva A, Elli S, Cosentino C, Torri G, Naggi A. Susceptibility of enoxaparin reducing end amino sugars to periodate oxidation. Carbohydr Res. (2014) 400:33-43. doi: 10.1016/j.carres.2014.08.016

36. Casu B, Guerrini M, Guglieri S, Naggi A, Perez M, Torri G, et al. Undersulfated and glycol-split heparins endowed with antiangiogenic activity. J Med Chem. (2004) 47:838-48. doi: 10.1021/jm030893g

37. Zong C, Venot A, Li X, Lu W, Xiao W, Wilkes JL, et al. Heparan sulfate microarray reveals that heparan sulfate-protein binding exhibits different ligand requirements. J Am Chem Soc. (2017) 139:9534-43. doi: 10.1021/jacs.7b01399

38. Seelen MA, Roos A, Wieslander J, Mollnes TE, Sjoholm AG, Wurzner R, et al. Functional analysis of the classical, alternative, and MBL pathways of the complement system: standardization and validation of a simple ELISA. $J$ Immunol Methods. (2005) 296:187-98. doi: 10.1016/j.jim.2004.11.016

39. Roos A, Bouwman LH, Munoz J, Zuiverloon T, Faber-Krol MC, Fallauxvan den Houten FC, et al. Functional characterization of the lectin pathway of complement in human serum. Mol Immunol. (2003) 39:65568. doi: 10.1016/S0161-5890(02)00254-7

40. Petersen SV, Thiel S, Jensen L, Steffensen R, Jensenius JC. An assay for the mannan-binding lectin pathway of complement activation. J Immunol Methods. (2001) 257:107-16. doi: 10.1016/S0022-1759(01)00453-7

41. Ambrus G, Gal P, Kojima M, Szilagyi K, Balczer J, Antal J, et al. Natural substrates and inhibitors of mannan-binding lectin-associated serine protease-1 and-2: a study on recombinant catalytic fragments. J Immunol. (2003) 170:1374-82. doi: 10.4049/jimmunol.170.3.1374

42. Seffouh A, El Masri R, Makshakova O, Gout E, Hassoun ZEO, Andrieu JP, et al. Expression and purification of recombinant extracellular sulfatase HSulf-2 allows deciphering of enzyme sub-domain coordinated role for the binding and 6-O-desulfation of heparan sulfate. Cell Mol Life Sci. (2019) 76:1807-19. doi: 10.1007/s00018-019-03027-2

43. van den Born J, van den Heuvel LP, Bakker MA, Veerkamp JH, Assmann KJ, Weening JJ, et al. Distribution of GBM heparan sulfate proteoglycan core protein and side chains in human glomerular diseases. Kidney Int. (1993) 43:454-63. doi: 10.1038/ki.1993.67

44. Sasisekharan R, Venkataraman G. Heparin and heparan sulfate: biosynthesis, structure and function. Curr Opin Chem Biol. (2000) 4:626-31. doi: 10.1016/S1367-5931(00)00145-9

45. Hein E, Honore C, Skjoedt MO, Munthe-Fog L, Hummelshoj T, Garred P. Functional analysis of ficolin-3 mediated complement activation. PLOS ONE. (2010) 5:e15443. doi: 10.1371/journal.pone.0015443

46. Heja D, Kocsis A, Dobo J, Szilagyi K, Szasz R, Zavodszky P, et al. Revised mechanism of complement lectin-pathway activation revealing the role of serine protease MASP-1 as the exclusive activator of MASP-2. Proc Natl Acad Sci USA. (2012) 109:10498-503. doi: 10.1073/pnas.1202588109

47. Zaferani A, Talsma D, Richter MK, Daha MR, Navis GJ, Seelen MA, et al. Heparin/heparan sulphate interactions with complement-a possible target for reduction of renal function loss? Nephrol Dial Transplant. (2014) 29:51522. doi: $10.1093 / \mathrm{ndt} / \mathrm{gft} 243$

48. Caughman GB, Boackle RJ, Vesely J. A postulated mechanism for heparin's potentiation of C1 inhibitor function. Mol Immunol. (1982) 19:28795. doi: 10.1016/0161-5890(82)90342-X

49. Dobo J, Harmat V, Beinrohr L, Sebestyen E, Zavodszky P, Gal P. MASP1, a promiscuous complement protease: structure of its catalytic region reveals the basis of its broad specificity. J Immunol. (2009) 183:120714. doi: 10.4049/jimmunol.0901141

50. Kjaer TR, Thiel S, Andersen GR. Toward a structure-based comprehension of the lectin pathway of complement. Mol Immunol. (2013) 56:41322. doi: 10.1016/j.molimm.2013.05.007

51. Duncan RC, Mohlin F, Taleski D, Coetzer TH, Huntington JA, Payne RJ, et al. Identification of a catalytic exosite for complement component C4 on the serine protease domain of C1s. J Immunol. (2012) 189:236573. doi: 10.4049/jimmunol.1201085

52. Kidmose RT, Laursen NS, Dobo J, Kjaer TR, Sirotkina S, Yatime L, et al. Structural basis for activation of the complement system by component C4 cleavage. Proc Natl Acad Sci USA. (2012) 109:1542530. doi: 10.1073/pnas.1208031109

53. Thompson JD, Higgins DG, Gibson TJ. CLUSTAL W: improving the sensitivity of progressive multiple sequence alignment through sequence weighting, position-specific gap penalties and weight matrix choice. Nucleic Acids Res. (1994) 22:4673-80. doi: 10.1093/nar/22.22.4673

54. Keizer MP, Pouw RB, Kamp AM, Patiwael S, Marsman G, Hart MH, et al. TFPI inhibits lectin pathway of complement activation by direct interaction with MASP-2. Eur J Immunol. (2015) 45:544-50. doi: 10.1002/eji.201445070

55. Peraramelli S, Thomassen S, Heinzmann A, Hackeng TM, Hartmann R, Scheiflinger F, et al. Role of exosite binding modulators in the inhibition of Fxa by TFPI. Thromb Haemost. (2016) 115:580-90. doi: 10.1160/th15-04-0354

56. Szakacs D, Kocsis A, Szasz R, Gal P, Pal G. Novel MASP-2 inhibitors developed via directed evolution of human TFPI1 are potent lectin pathway inhibitors. $J$ Biol Chem. (2019) 294:8227-37. doi: 10.1074/jbc.RA119.008315

Conflict of Interest: The authors declare that the research was conducted in the absence of any commercial or financial relationships that could be construed as a potential conflict of interest.

Copyright (C) 2020 Talsma, Poppelaars, Dam, Meter-Arkema, Vivès, Gál, Boons, Chopra, Naggi, Seelen, Berger, Daha, Stegeman, van den Born and the COMBAT Consortium. This is an open-access article distributed under the terms of the Creative Commons Attribution License (CC BY). The use, distribution or reproduction in other forums is permitted, provided the original author(s) and the copyright owner(s) are credited and that the original publication in this journal is cited, in accordance with accepted academic practice. No use, distribution or reproduction is permitted which does not comply with these terms. 